\title{
Support varieties and cohomology over complete intersections
}

\author{
Luchezar L. Avramov ${ }^{1, \star}$, Ragnar-Olaf Buchweitz ${ }^{2, \star \star}$ \\ 1 Department of Mathematics, Purdue University, West Lafayette, IN 47907, USA \\ (e-mail: avramov@math.purdue.edu) \\ 2 Department of Mathematics, University of Toronto, Toronto, ON M5S 3G3, Canada \\ (e-mail: ragnar@math.utoronto.ca)
}

Oblatum 23-XII-1999 \& 28-III-2000

Published online: 5 June 2000 - (C) Springer-Verlag 2000

\section{Introduction}

Quillen's geometric approach to the cohomology of finite groups [30] has revolutionized modular representation theory. The ideology and techniques involved have been generalized and extended to representations of various Hopf algebras, culminating in the recent work of Friedlander and Suslin [17] on finite group schemes.

In this paper we develop geometric methods for the study of finite modules over a local complete intersection $R$. If $R$ is such a ring and $\mathfrak{m}$ is its maximal ideal, then the $\mathfrak{m}$-adic completion $\widehat{R}$ has the form $Q /(f)$, where $f$ is a regular sequence and $Q$ is a regular local ring that can be taken to be a ring of formal power series over a field or a discrete valuation ring. The least number of equations needed to cut out $\widehat{R}$ from a regular local ring equals the codimension of $R$, where $\operatorname{codim} R=v_{R}(\mathfrak{m})-\operatorname{dim} R$ and $v_{R}(M)$ denotes the minimal number of generators of an $R$-module $M$.

In [5] a cone, that is, a homogeneous algebraic set $\mathrm{V}_{R}^{*}(M)$ is attached to each finite $R$-module $M$ and used to study its minimal free resolution. Here we prove

Theorem I. If $R$ is a local complete intersection of codimension $c$, and $\widetilde{k}$ is an algebraic closure of the residue field $k$ of $R$, then for any two finite $R$-modules $M, N$ there exists a cone $\mathrm{V}_{R}^{*}(M, N) \subseteq \widetilde{k}^{c}$, such that

^ L.L.A. was partly supported by a grant from the NSF.

$\star \star$ R.O.B. was partly supported by a grant from NSERC.

Mathematics Subject Classification (1991): 13D05, 13C40, 14M10, 18G15, 20 J06 
(1) $\mathrm{V}_{R}^{*}(M, M)=\mathrm{V}_{R}^{*}(M, k)=\mathrm{V}_{R}^{*}(k, M)=\mathrm{V}_{R}^{*}(M)$.

(2) $\mathrm{V}_{R}^{*}(M, N)=\{0\}$ if and only if $\operatorname{Ext}_{R}^{n}(M, N)=0$ for $n \gg 0$.

(3) $\mathrm{V}_{R}^{*}(M, N)=\mathrm{V}_{R}^{*}(M) \cap \mathrm{V}_{R}^{*}(N)=\mathrm{V}_{R}^{*}(N, M)$.

The first assertion shows that the present theory agrees with and extends that of [5]. We also complement the earlier results in an important way by exhibiting a set of polynomial equations defining $\mathrm{V}_{R}^{*}(M)$ that can be computed solely from a finite free resolution of $M$ over the regular ring $Q$.

The second assertion is the simplest manifestation of a general phenomenon: the dimension of the variety $\mathrm{V}_{R}^{*}(M, N)$ measures "the size" of $\operatorname{Ext}_{R}^{*}(M, N)$. To make this precise, we define the complexity of a pair of modules $(M, N)$ to be the number

$$
\operatorname{cx}_{R}(M, N)=\inf \left\{\begin{array}{l|l}
b \in \mathbb{N} & \begin{array}{c}
v_{R}\left(\operatorname{Ext}_{R}^{n}(M, N)\right) \leq a n^{b-1} \text { for some } \\
\text { real number } a \text { and for all } n \gg 0
\end{array}
\end{array}\right\} .
$$

This notion encompasses the asymptotic invariants of $M$ studied in [5]: its complexity $\mathrm{cx}_{R} M=\mathrm{cx}_{R}(M, k)$, measuring the polynomial rate of growth at infinity of its minimal free resolution, and its plexity $\operatorname{px}_{R} M=\operatorname{cx}_{R}(k, M)$, giving the corresponding information on its minimal injective resolution. Results of Shamash [31] and Gulliksen [21] show that neither number exceeds the codimension of $R$.

By construction, varieties of pairs satisfy $\operatorname{cx}_{R}(M, N)=\operatorname{dim~}_{R}^{*}(M, N)$. Thus, their properties listed in Theorem I immediately translate into numerical data.

Theorem II. For finite modules $M, N$ over a local complete intersection $R$, the following (in)equalities hold:

(1) $\operatorname{cx}_{R}(M, M)=\mathrm{cx}_{R} M=\mathrm{px}_{R} M$.

(2) $\operatorname{cx}_{R}(M, N)=0$ if and only if $\operatorname{Ext}_{R}^{n}(M, N)=0$ for $n \gg 0$.

(3) $\operatorname{cx}_{R} M+\operatorname{cx}_{R} N-\operatorname{codim} R \leq \operatorname{cx}_{R}(M, N)=\operatorname{cx}_{R}(N, M)$

$$
\leq \min \left\{\operatorname{cx}_{R} M, \operatorname{cx}_{R} N\right\} \text {. }
$$

We do not know any other proof of the relations above. In fact, we are not even aware of any earlier indication that such relations might hold.

For instance, (2) and (3) yield an unexpected and remarkable property of finite modules over a local complete intersection: (ee) $\operatorname{Ext}_{R}^{n}(M, N)=0$ for $n \gg 0$ if and only if $\operatorname{Ext}_{R}^{n}(N, M)=0$ for $n \gg 0$. We prove a more precise global version, that also characterizes the eventual vanishing of $\operatorname{Tor}_{n}^{R}(M, N)$. Denoting $\operatorname{Max}(R)$ the set of maximal ideals of $R$, we can state a form of the result as follows:

Theorem III. Let $R$ be a commutative noetherian ring such that the local ring $R_{\mathfrak{m}}$ is a complete intersection for each maximal ideal $\mathfrak{m}$ of $R$, assume that the Krull dimension $\operatorname{dim} R=d$ is finite, and set $c=\sup \left\{\operatorname{codim} R_{\mathfrak{m}} \mid\right.$ $\mathfrak{m} \in \operatorname{Max}(R)\}$. 
For finite R-modules $M, N$ the following conditions are equivalent.

(i) $\operatorname{Ext}_{R}^{n}(M, N)=0$ for $h \leq n \leq h+c$ and some $h>d$.

(ii) $\operatorname{Ext}_{R}^{n}(N, M)=0$ for $i \leq n \leq i+c$ and some $i>d$.

(iii) $\operatorname{Tor}_{n}^{R}(M, N)=0$ for $j \leq n \leq j+c$ and some $j \geq 0$.

(iv) $\operatorname{Ext}_{R}^{n}(M, N)=\operatorname{Ext}_{R}^{n}(N, M)=\operatorname{Tor}_{n}^{R}(M, N)=0$ for $n>d$.

It is easy to see that any commutative noetherian $\operatorname{ring} R$ with property (ee) is Gorenstein. By our result, the class of rings satisfying (ee) is sandwiched between the class of locally complete intersection rings and that of Gorenstein rings. We do not know whether it is equal to either of those classes.

There are two aspects to the proof of the last theorem. One is to show that "long" gaps in a sequence of (co)homology modules force it to vanish beyond $\operatorname{dim} R$; this is handled by standard homological algebra, and is known for Tor from work of Murthy [28]. To compare asymptotic vanishing of Ext's and Tor's, we establish that the latter is also governed by the intersection of the varieties of $M$ and $N$.

Theorem IV. If $M, N$ are finite modules over a local complete intersection $R$, then $\operatorname{Tor}_{n}^{R}(M, N)=0$ for $n \gg 0$ if and only if $\mathrm{V}_{R}^{*}(M) \cap \mathrm{V}_{R}^{*}(N)=\{0\}$.

The theorem answers a question of Jorgensen [23], who proved the 'only if' part. It also extends to arbitrary codimension a result of Huneke and R. Wiegand [22]: If $R$ is a hypersurface, then $\operatorname{Tor}_{n}^{R}(M, N)=0$ for $n \gg 0$ (if and) only if $M$ or $N$ has finite projective dimension; indeed, a cone in $\widetilde{k}^{1}$ is either equal to $\widetilde{k}^{1}$ itself, or is reduced to $\{0\}$, and for $\mathrm{V}_{R}^{*}(M)$ the second case means $\operatorname{proj}_{\operatorname{dim}} M<\infty$.

The proofs of the theorems discussed above are given in Sects. 5 and 6, drawing on more general results established in the intervening sections. In particular, we prove and use a concise, versatile vanishing result in terms of stable (co)homology.

Many results on varieties over complete intersections have prototypes over group algebras, cf. [10] for an exposition, but the analogy should be used with care. For instance, the intersection formula in Theorem I fails over group algebras: Benson, Carlson, and Robinson [11] show that certain groups have non-projective modules $N$ with $\mathrm{H}^{n}(G, N)=0$ for $n>0$, whence $\{0\}=\mathrm{V}_{G}^{*}(k, N) \subsetneq \mathrm{V}_{G}^{*}(k) \cap \mathrm{V}_{G}^{*}(N)=\mathrm{V}_{G}^{*}(N)$.

A difference from the situation over group algebras is the absence of a Hopf algebra structure, so that there is no obvious ring of operators over which all cohomology in sight would be finitely generated. Patching together different actions on $\operatorname{Ext}_{R}^{*}(M, N)$ is an important and non-trivial problem. Another basic difference is that modules over local rings are not finitedimensional vector spaces, so the use of duality is restricted and delicate. 
As a result, the methods we develop are often quite unlike those used for groups.

The geometric study of cohomology over commutative local rings $R$ is not limited to complete intersections. In fact, we establish many results in the more general context of modules of finite CI-dimension, introduced in [8]. However, there are known obstacles to extend the geometric approach to arbitrary finite modules over local rings. For example, if the localization $R_{\mathfrak{p}}$ at a prime ideal $\mathfrak{p}$ is not a complete intersection, then the $R$-module $R / \mathfrak{p}$ has infinite complexity - in fact, its minimal free resolution grows exponentially, cf. [6].

\section{Cohomology operators}

A major role in our investigation is played by an action on $\operatorname{Ext}_{R}^{*}(M, N)$ of a graded polynomial ring, introduced by Gulliksen in [21] and studied by Eisenbud, Gasharov, Mehta, Peeva, Sun, and the authors, cf. [5], [7], [8], [9], [16], [26].

1.1. Let $\boldsymbol{f}=f_{1}, \ldots, f_{c}$ be a regular sequence in a commutative ring $Q$, and let $R=Q /(\boldsymbol{f})$. For all $R$-modules $M, N$, Gulliksen [21] defined on the graded $R$-module $\operatorname{Ext}_{R}^{*}(M, N)$ a structure of graded module over a polynomial ring of cohomology operators $\delta=R\left[\chi_{1}, \ldots, \chi_{c}\right]$ with variables of degree 2 , so that

$$
\chi_{j}: \operatorname{Ext}_{R}^{n}(M, N) \rightarrow \operatorname{Ext}_{R}^{n+2}(M, N) \text { for } n \in \mathbb{Z} \quad \text { and } j=1, \ldots, c .
$$

Various constructions of that $\&$-module structure are needed to prove the following properties of cohomology operators, but by $[9, \S 4]$ they all coincide up to sign.

1.1.1. (Eisenbud [16, (1.7)], cf. also [9, (3.1)].) Consider a commutative diagram

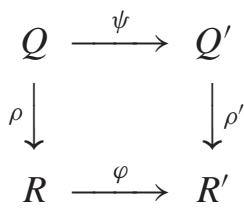

of ring homomorphisms with $\operatorname{Ker} \rho^{\prime}=\left(\boldsymbol{f}^{\prime}\right)$, where $\boldsymbol{f}^{\prime}=f_{1}^{\prime}, \ldots, f_{c^{\prime}}^{\prime}$ and

$$
\psi\left(f_{i}\right)=\sum_{j=1}^{c^{\prime}} \psi\left(q_{i j}\right) f_{j}^{\prime} \quad \text { with } \quad q_{i j} \in Q \quad \text { for } \quad 1 \leq i \leq c .
$$

If $\boldsymbol{f}^{\prime}$ is $Q^{\prime}$-regular, if $\chi_{1}^{\prime}, \ldots, \chi_{c^{\prime}}^{\prime}$ is the family of cohomology operators it defines, $M^{\prime}, N^{\prime}$ are $R^{\prime}$-modules, and $\mu: M \rightarrow M^{\prime}, v: N^{\prime} \rightarrow N$ are $R$-linear 
maps, then

$$
\sum_{i=1}^{c} q_{i j} \chi_{i} \circ \operatorname{Ext}_{\varphi}^{*}(\mu, v)=\operatorname{Ext}_{\varphi}^{*}(\mu, \nu) \circ \chi_{j}^{\prime} \quad \text { for } \quad j=1, \ldots, c^{\prime}
$$

where $\operatorname{Ext}_{\varphi}^{*}(\mu, v)$ is the canonical map $\operatorname{Ext}_{R^{\prime}}^{*}\left(M^{\prime}, N^{\prime}\right) \rightarrow \operatorname{Ext}_{R}^{*}(M, N)$.

1.1.2. (Mehta [26, §2]; cf. also [9, (3.3)].) Composition products on Ext modules, denoted . , turn $\operatorname{Ext}_{R}^{*}(M, M)$ and $\operatorname{Ext}_{R}^{*}(N, N)$ into graded $R$ algebras, and $\operatorname{Ext}_{R}^{*}(M, N)$ into a graded left $\operatorname{Ext}_{R}^{*}(N, N)$-, right $\operatorname{Ext}_{R}^{*}(M, M)$ bimodule. There exist canonical homomorphisms of graded $R$-algebras

$$
\operatorname{Ext}_{R}^{*}(M, M) \stackrel{\xi_{M}}{\longleftarrow} \stackrel{\xi_{N}}{\longrightarrow} \operatorname{Ext}_{R}^{*}(N, N)
$$

such that for all $\gamma \in \operatorname{Ext}_{R}^{*}(M, N)$ there are equalities

$$
\xi_{N}\left(\chi_{j}\right) \cdot \gamma=\chi_{j} \gamma=\gamma \cdot \xi_{M}\left(\chi_{j}\right) \quad \text { for } \quad j=1, \ldots, c \text {. }
$$

Composition products are functorial in both module arguments and commute with connecting homomorphisms, hence the action of $\delta$ shares these properties.

1.1.3. (Gulliksen $[21,(3.1)]$; cf. also [5, (2.1)], [7, (6.2)].) If the graded $R$ module $\operatorname{Ext}_{Q}^{*}(M, N)$ is noetherian, then the graded $\&$-module $\operatorname{Ext}_{R}^{*}(M, N)$ is noetherian.

1.1.4. (Avramov-Gasharov-Peeva [8, (4.2)].) If the graded $\&$-module $\operatorname{Ext}_{R}^{*}(M, N)$ is noetherian, then the graded $R$-module $\operatorname{Ext}_{Q}^{*}(M, N)$ is noetherian.

For illustration and for later use, we present a computation of the action of cohomology operators, using a technique from [7].

If $\mathcal{N}$ is a graded $\delta$-module, then $\mathcal{N}\{p\}$ denotes the graded module with $\mathcal{N}\{p\}^{q}=\mathcal{N}^{q-p}$, and $\mathcal{K}\left(v_{1}, \ldots, v_{b} ; \mathcal{N}\right)$ is the Koszul complex on a set $v_{1}, \ldots, v_{b} \in \&$, with differential of cohomological degree 1 and $\mathcal{K}_{p}$ in complex degree $-p$.

1.2. Proposition. Given a commutative diagram of ring homomorphisms 1.1.1 with $\psi=\mathrm{id}_{Q}$ and an $R^{\prime}$-algebra $S$, there is an isomorphism of graded s-modules

$$
\operatorname{Ext}_{R}^{\mathrm{par}}\left(R^{\prime}, S\right) \cong \bigoplus_{p, p \text { par }} \mathscr{H}^{-p}\left(\mathcal{K}\left(v_{1}, \ldots, v_{c^{\prime}} ; S\left[\chi_{1}, \ldots, \chi_{c}\right]\right)\right)\{p\}
$$

where par stands for either even or odd, and $v_{i}=\sum_{j=1}^{c} q_{i j} \chi_{j}$ for $i=$ $1, \ldots, c^{\prime}$. 
Proof. Let $\boldsymbol{K}$ and $\boldsymbol{K}^{\prime}$ be the Koszul complexes that resolve $R=Q /(\boldsymbol{f})$ and $R^{\prime}=Q /\left(f^{\prime}\right)$, respectively, over $Q$. Choosing bases $\xi_{1}, \ldots, \xi_{c}$ of $K_{1}$ and $\xi_{1}^{\prime}, \ldots, \xi_{c^{\prime}}^{\prime}$ of $K_{1}^{\prime}$, with $\partial\left(\xi_{j}\right)=f_{j}$ for $j=1, \ldots, c$ and $\partial\left(\xi_{i}^{\prime}\right)=f_{i}^{\prime}$ for $i=1, \ldots, c^{\prime}$, we define a morphism of DG algebras $\phi: K \rightarrow \boldsymbol{K}^{\prime}$ by $\phi\left(\xi_{j}\right)=\sum_{i=1}^{c^{\prime}} q_{i j} \xi_{i}^{\prime}$ for $j=1, \ldots, c$.

The differential of $A=\operatorname{Hom}_{Q}\left(\boldsymbol{K}^{\prime}, S\right)$ is trivial, so $\operatorname{Ext}_{Q}^{*}\left(R^{\prime}, S\right)=A$. Let $\left\{\zeta_{\boldsymbol{h}}\right\}$, where $\boldsymbol{h}=\left(h_{1}, \ldots, h_{p}\right) \in \mathbb{N}^{p}$ with $h_{1}<\cdots<h_{p}$, be the $S$-basis of $A^{p}=\operatorname{Hom}_{Q}\left(K_{p}^{\prime}, S\right)$ dual to the basis $\left\{\xi_{h_{1}}^{\prime} \wedge \cdots \wedge \xi_{h_{p}}^{\prime}\right\}$ of $K_{p}^{\prime} \otimes_{Q} S$. By [7, (1.9)] the graded algebra $\boldsymbol{K}^{\prime} \otimes_{Q} S$ acts on $A=\operatorname{Ext}_{Q}^{*}\left(R^{\prime}, S\right)$ by $\xi_{i}^{\prime} \zeta_{\boldsymbol{h}}=(-1)^{p+r-1} \zeta_{\boldsymbol{h} \backslash h_{r}}$ if $i=h_{r} \in \boldsymbol{h}$ and $\xi_{i}^{\prime} \zeta_{\boldsymbol{h}}=0$ if $i \notin \boldsymbol{h}$. From [7, (3.7)] we conclude that

$$
\operatorname{Ext}_{R}^{\mathrm{par}}\left(R^{\prime}, S\right) \cong \bigoplus_{p, p \text { par }} \mathcal{H}^{-p}\left(\mathcal{C}^{\bullet}\left(R^{\prime}, S\right)\right)\{p\}
$$

as graded $\&$-modules, where $\mathcal{C} \bullet\left(R^{\prime}, S\right)$ is the complex of graded $\&$-modules

$$
\begin{aligned}
& \ldots \longrightarrow \& \otimes_{R} A^{p+1}\{p+1\} \longrightarrow \mathrm{d} \\
& \stackrel{\mathrm{d}}{\longrightarrow} \& \otimes_{R} A^{p-1}\{p-1\} \longrightarrow \ldots
\end{aligned}
$$

with $s \otimes_{R} A^{p}\{p\}$ sitting in complex degree $-p$ and differential given by

$$
\begin{aligned}
\mathrm{d}\left(\chi \otimes \zeta_{\boldsymbol{h}}\right) & =\sum_{j=1}^{c} \chi_{j} \chi \otimes \sum_{i=1}^{c^{\prime}} q_{i j} \xi_{i}^{\prime} \zeta_{\boldsymbol{h}} \\
& =(-1)^{p} \sum_{h_{r} \in \boldsymbol{h}}\left(\sum_{j=1}^{c} q_{i j} \chi_{j} \chi\right)(-1)^{r-1} \otimes \zeta_{\boldsymbol{h} \backslash h_{r}} .
\end{aligned}
$$

It is clear that $\mathcal{C}^{\bullet}\left(R^{\prime}, S\right)$ is isomorphic to the desired Koszul complex.

The concept of complexity used below is defined in the introduction.

1.3. Proposition. Let $Q$ be a noetherian local ring with unique maximal ideal $\mathfrak{n}, \boldsymbol{f}=f_{1}, \ldots, f_{c}$ a $Q$-regular sequence, and $M, N$ finite modules over $R=Q /(\boldsymbol{f})$.

If $\operatorname{Ext}_{Q}^{n}(M, N)=0$ for $n \gg 0$, then $\mathscr{E}=\operatorname{Ext}_{R}^{*}(M, N) \otimes_{R} k$ is a finite graded module over the graded polynomial ring $\mathcal{R}=\varsigma \otimes_{R} k=$ $k\left[\chi_{1}, \ldots, \chi_{c}\right]$. When $\& \neq 0$ the Krull dimension $\operatorname{dim}_{\mathcal{R}} \&$ is equal to $\mathrm{cx}_{R}(M, N)$, and the formal power series

$$
\left(1-t^{2}\right)^{\operatorname{cx}_{R}(M, N)} \sum_{n=0}^{\infty} v_{R}\left(\operatorname{Ext}_{R}^{n}(M, N)\right) t^{n}
$$

is a polynomial with integer coefficients that has no root at $t=1$. 
Proof. It follows immediately from 1.1.3 that $\mathcal{E}$ is a finite module over $\mathcal{R}=\delta \otimes_{R} k$, which is itself a polynomial ring in $c$ variables of degree 2 over the field $k$. Thus, $\mathcal{E}$ is the direct sum $\mathcal{E}^{\text {even }} \oplus \mathcal{E}^{\text {odd }}$ of its submodules consisting of elements of even and odd degree, respectively. In particular, $\operatorname{dim}_{\mathcal{R}} \mathcal{E}=\max \left\{\operatorname{dim}_{\mathcal{R}} \mathcal{E}^{\text {even }}, \operatorname{dim}_{\mathcal{R}} \mathcal{E}^{\text {odd }}\right\}$.

By the Hilbert-Serre Theorem, $\sum_{n=0}^{\infty} \operatorname{rank}_{k} \mathcal{E}^{n} t^{n}$ represents a rational function with denominator $\left(1-t^{2}\right)^{d}$, where $d=\operatorname{dim}_{\mathcal{R}} \mathcal{E}$, and numerator that does not vanish at $t=1$. It is well known, and easily seen from a decomposition into partial fractions, that $d$ is the least integer such that $\operatorname{rank}_{k} \mathcal{E}^{n} \leq a n^{d-1}$ for some real number $a$ and all $n \gg 0$. Since $\operatorname{rank}_{k} \mathcal{E}^{n}=$ $v_{R}\left(\operatorname{Ext}_{R}^{n}(M, N)\right)$, we are done.

A special case of the proposition, cf. [21, (4.2)], deals with the series

$$
\mathrm{P}_{M}^{R}(t)=\sum_{n=0}^{\infty} \operatorname{rank}_{k} \operatorname{Ext}_{R}^{n}(M, k) t^{n} \quad \text { and } \quad \mathrm{I}_{R}^{M}(t)=\sum_{n=0}^{\infty} \operatorname{rank}_{k} \operatorname{Ext}_{R}^{n}(k, M) t^{n}
$$

known, respectively, as the Poincaré series and the Bass series of $M$. By definition, $\mathrm{cx}_{R} M=\mathrm{cx}_{R}(M, k)$ and $\mathrm{px}_{R} M=\mathrm{cx}_{R}(k, N)$, where $k$ is the residue field of $R$.

1.4. Corollary. If proj $\operatorname{dim}_{Q} M<\infty$, then $\mathrm{cx}_{R} M \leq$ and $\left(1-t^{2}\right)^{\mathrm{cx}_{R} M} \mathrm{P}_{M}^{R}(t)$ is a polynomial with integer coefficients that does not vanish at $t=1$ when $M \neq 0$.

If inj $\operatorname{dim}_{Q} M<\infty$, then $\mathrm{px}_{R} M \leq c$ and $\left(1-t^{2}\right)^{\mathrm{px}_{R} M} \mathrm{I}_{R}^{M}(t)$ is a polynomial with integer coefficients that does not vanish at $t=1$ when $M \neq 0$.

The next result and its proof are cohomological versions of results on modules with eventually vanishing Tor's, proved by C. Miller [27, (2.1), (2.2)].

1.5. Proposition. Let $R$ be a local ring with residue field $k$, and let $M, N$ be finite $R$-modules such that $\operatorname{Ext}_{R}^{n}(M, N)=0$ for $n>0$. The following then hold.

(1) $\mathrm{I}_{R}^{\mathrm{Hom}_{R}(M, N)}(t)=\mathrm{P}_{M}^{R}(t) \mathrm{I}_{R}^{N}(t)$.

(2) $\operatorname{px}_{R} \operatorname{Hom}_{R}(M, N) \leq \mathrm{cx}_{R} M+\mathrm{px}_{R} N$.

(3) If furthermore $R=Q /(\boldsymbol{f})$ for a local ring $Q$ and a regular se-

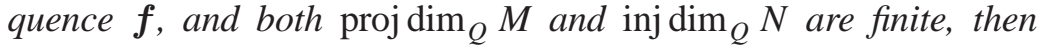
equality holds in (2).

Proof. (1) Let $\boldsymbol{F}$ be a free resolution of $M$, indexed by lower degrees, and $\boldsymbol{J}$ an injective resolution of $N$, indexed by upper degrees. The complex $\operatorname{Hom}_{R}(\boldsymbol{F}, \boldsymbol{J})$ has the injective module $\prod_{i=0}^{n} \operatorname{Hom}\left(F_{i}, J^{n-i}\right)$ as component 
of upper degree $n$, and

$$
\mathrm{H}^{n}\left(\operatorname{Hom}_{R}(\boldsymbol{F}, \boldsymbol{J})\right) \cong \operatorname{Ext}_{R}^{n}(M, N)= \begin{cases}\operatorname{Hom}_{R}(M, N) & \text { if } n=0 \\ 0 & \text { if } n>0\end{cases}
$$

Computing with this injective resolution of $\operatorname{Hom}_{R}(M, N)$, we have

$$
\begin{aligned}
\operatorname{Ext}_{R}^{n}\left(k, \operatorname{Hom}_{R}(M, N)\right) & =\mathrm{H}^{n} \operatorname{Hom}_{R}\left(k, \operatorname{Hom}_{R}(\boldsymbol{F}, \boldsymbol{J})\right) \\
& \cong \mathrm{H}^{n} \operatorname{Hom}_{R}\left(k \otimes_{R} \boldsymbol{F}, \boldsymbol{J}\right) \\
& \cong \mathrm{H}^{n} \operatorname{Hom}_{k}\left(k \otimes_{R} \boldsymbol{F}, \operatorname{Hom}_{R}(k, \boldsymbol{J})\right) \\
& \cong \prod_{i=0}^{n} \operatorname{Hom}_{k}\left(\mathrm{H}_{i}\left(k \otimes_{R} \boldsymbol{F}\right), \mathrm{H}^{n-i} \operatorname{Hom}_{R}(k, \boldsymbol{J})\right) \\
& \cong \prod_{i=0}^{n} \operatorname{Hom}_{k}\left(\operatorname{Tor}_{i}^{R}(k, M), \operatorname{Ext}_{n}^{n-i}(k, N)\right) .
\end{aligned}
$$

Counting ranks of $k$-vector spaces, for $n \geq 0$ we get numerical equalities $\operatorname{rank}_{k} \operatorname{Ext}_{R}^{n}\left(k, \operatorname{Hom}_{R}(M, N)\right)=\sum_{i=0}^{n} \operatorname{rank}_{k} \operatorname{Tor}_{i}^{R}(k, M) \cdot \operatorname{rank}_{k} \operatorname{Ext}_{R}^{n-i}(k, N)$.

This is just another way to write the announced equality of power series.

(2) The desired inequality follows from the equality of power series in (1).

(3) By Proposition 1.3 and Corollary 1.4 the Bass series of $\operatorname{Hom}_{R}(M, N)$, the Poincare series of $M$, and the Bass series of $N$ represent rational functions of $t$ having at $t=1$ poles of order $\operatorname{px}_{R} \operatorname{Hom}_{R}(M, N), \mathrm{cx}_{R} M$, and $\mathrm{px}_{R} N$, respectively. Equating the orders of the poles on both sides of (1) we get the desired equality.

\section{Support varieties}

In this section $Q$ is a commutative noetherian local ring with maximal ideal $\mathfrak{n}$ and residue field $k=Q / \mathfrak{n}$, and $\widetilde{k}$ is an algebraic closure of $k$. Further, $R=Q / \mathfrak{a}$ where $\mathfrak{a}$ is generated by a $Q$-regular sequence $\boldsymbol{f}=f_{1}, \ldots, f_{c}$ and $\delta=R\left[\chi_{1}, \ldots, \chi_{c}\right]$ is the ring of cohomology operators defined by $f$ and $\mathcal{R}=s \otimes_{R} k=k\left[\chi_{1}, \ldots, \chi_{c}\right]$.

2.1. The support variety $\mathrm{V}(Q, f ; M, N)$ of a pair of finite $R$-modules $(M, N)$ is the zero set in $\widetilde{k}^{c}$ of the annihilator of $\mathcal{E}=\operatorname{Ext}_{R}^{*}(M, N) \otimes_{R} k$ in $\mathcal{R}$, that is

$$
\mathrm{V}(Q, \boldsymbol{f} ; M, N)=\left\{\begin{array}{l|l}
\left(b_{1}, \ldots, b_{c}\right) \in \widetilde{k}^{c} & \begin{array}{l}
\phi\left(b_{1}, \ldots, b_{c}\right)=0 \\
\text { for all } \phi \in \operatorname{ann}_{\mathcal{R}} \mathcal{E}
\end{array}
\end{array}\right\} \cup\{0\} .
$$


As $\mathscr{E}$ is graded, its annihilator is a homogeneous ideal, so $\mathrm{V}(Q, f ; M, N)$ is a cone.

The variety does not depend on the choice of regular sequence generating $\mathfrak{a}$. To see this, and for later use, it is convenient to adjust the residue field of a local ring.

By a residual algebraic closure of $Q$ we mean a flat extension of local rings $Q \subseteq \widetilde{Q}$ such that $\mathfrak{n} \widetilde{Q}$ is the maximal ideal $\widetilde{\mathfrak{n}}$ of $\widetilde{Q}$ and the induced map $k \rightarrow \widetilde{Q} / \widetilde{\mathfrak{n}}$ is the embedding of $k$ into an algebraic closure $\widetilde{k}$; such extensions always exist: one possible construction is by inflation, as in [12, App., Théorème 1, Corollaire].

2.2. Lemma. If $Q \subseteq \widetilde{Q}$ is a residual algebraic closure then $f$ is a $\widetilde{Q}$ regular sequence, the induced map $R \rightarrow \widetilde{R}=\widetilde{Q} /(\boldsymbol{f})$ is a residual algebraic closure, and

$$
\mathrm{V}(Q, \boldsymbol{f} ; M, N)=\mathrm{V}(\widetilde{Q}, \boldsymbol{f} ; \tilde{M}, \tilde{N}) \subseteq \widetilde{k}^{c}
$$

where $\widetilde{M}$ and $\widetilde{N}$ denote the $\widetilde{R}$-modules $M \otimes_{R} \widetilde{R}$ and $N \otimes_{R} \widetilde{R}$, respectively.

Proof. The sequence $f$ is $\widetilde{Q}$-regular because the inclusion $\psi: Q \subseteq \widetilde{Q}$ is flat. By 1.1.1 applied to $\varphi=\psi \otimes_{Q} \widetilde{Q}: R \subseteq \widetilde{R}$, the $c \times c$ identity matrix $\left(q_{i j}\right)$, and the $R$-linear maps $\mu: M \rightarrow \tilde{M}, \mu(m)=m \otimes 1$, and $v=\mathrm{id}_{\tilde{N}}$, we see that the map

$$
\operatorname{Ext}_{\varphi}^{*}(\mu, v): \operatorname{Ext}_{\widetilde{R}}^{*}(\tilde{M}, \tilde{N}) \longrightarrow \operatorname{Ext}_{R}^{*}(M, \tilde{N})
$$

of graded modules is compatible with the isomorphism $s \otimes_{R} \widetilde{R} \cong \widetilde{\S}$ of graded $\widetilde{R}$-algebras. As $\widetilde{R}$ is $R$-flat, both $\operatorname{Ext}_{\varphi}^{*}(\mu, v)$ and the canonical map of graded modules

$$
\operatorname{Ext}_{R}^{*}(M, N) \otimes_{R} \widetilde{R} \longrightarrow \operatorname{Ext}_{R}^{*}(M, \widetilde{N}) .
$$

are bijective. The desired equality of varieties is an immediate consequence.

2.3. Remark. The basis of $\mathfrak{a} / \mathfrak{n a}$ provided by the image of $\boldsymbol{f}$ yields an isomorphism $k^{c} \cong \mathfrak{a} / \mathfrak{n a}$ that extends to a $\widetilde{k}$-linear isomorphism $\alpha: \widetilde{k}^{c} \cong$ $\widetilde{Q} \mathfrak{a} / \tilde{\mathfrak{n}} \mathfrak{a}$. If $\boldsymbol{f}^{\prime}$ is a $Q$-regular sequence with $\left(\boldsymbol{f}^{\prime}\right)=\mathfrak{a}$, and $\alpha^{\prime}: \widetilde{k}^{c} \cong \widetilde{Q} \mathfrak{a} / \tilde{\mathfrak{n}} \mathfrak{a}$ is the corresponding isomorphism, then 1.1.1 applied to the identity maps of $Q, R, M$, and $N$ yields an isomorphism of $k$-algebras $\delta \cong R\left[\chi_{1}^{\prime}, \ldots, \chi_{c}^{\prime}\right]$ that is compatible with the identity map of $\mathcal{E}$, and hence $\alpha^{\prime}\left(\mathrm{V}\left(Q, \boldsymbol{f}^{\prime} ; M, N\right)\right)=$ $\alpha(\mathrm{V}(Q, \boldsymbol{f} ; M, N))$.

Thus, we have a well defined algebraic subset $\mathrm{V}(Q, R ; M, N) \subseteq$ $\widetilde{Q} \mathfrak{a} / \widetilde{\mathfrak{n}} \mathfrak{a}$. 
2.4. Proposition. For any two R-modules $M, N$ the following holds.

(1) $\mathrm{V}(Q, \boldsymbol{f} ; M, N)=\{0\}$ if and only if $\operatorname{Ext}_{R}^{n}(M, N)=0$ for $n \gg 0$.

If furthermore $\operatorname{Ext}_{Q}^{n}(M, N)=0$ for $n \gg 0$, then the following hold.

(2) $\operatorname{dim} \mathrm{V}(Q, \boldsymbol{f} ; M, N)=\operatorname{cx}_{R}(M, N) \leq c$.

(3) $\mathrm{V}\left(Q, \boldsymbol{f} ; M^{\prime}, N^{\prime}\right)=\mathrm{V}(Q, \boldsymbol{f} ; M, N)$ if there exist exact sequences of $R$-modules

$$
\begin{aligned}
& 0 \rightarrow M^{\prime} \rightarrow F_{r} \rightarrow \cdots \rightarrow F_{0} \rightarrow M \rightarrow 0 \\
& 0 \rightarrow N^{\prime} \rightarrow G_{s} \rightarrow \cdots \rightarrow G_{0} \rightarrow N \rightarrow 0
\end{aligned}
$$

with $\operatorname{Ext}_{R}^{n}\left(F_{i}, N\right)=0=\operatorname{Ext}_{R}^{n}\left(M, G_{j}\right)$ for all $i, j$ and for $n \gg 0$.

Proof. (1) holds by the Nullstellensatz.

(2) The dimension of the algebraic set $\mathrm{V}(Q, \boldsymbol{f} ; M, N)$ is equal to the Krull dimension $\operatorname{dim}\left(\mathcal{R} / \operatorname{ann}_{\mathcal{R}} \mathcal{E}\right)$ by definition. By $1.1 .3, \mathcal{E}$ is a finite module over the polynomial ring in $c$ variables over $k$, so $\operatorname{dim}\left(\mathcal{R} / \operatorname{ann}_{\mathcal{R}} \mathcal{E}\right)=$ $\operatorname{dim}_{\mathcal{R}} \varepsilon \leq c$.

(3) Since $\operatorname{Ext}_{R}^{i}\left(F_{n}, N\right)=0$ for $n \gg 0$, the iterated connecting maps induced by the exact sequence linking $M^{\prime}$ and $M$ yield an isomorphism $\operatorname{Ext}_{R}^{\geqslant t^{\prime}}\left(M^{\prime}, N\right)\left[t^{\prime}\right] \cong \operatorname{Ext}_{R}^{\geqslant t}(M, N)[t]$, for appropriate $t, t^{\prime} \in \mathbb{Z}$. By 1.1.2 this isomorphism commutes with the actions of $\delta$, so the $\mathcal{R}$-modules $\operatorname{Ext}_{R}^{\geqslant t}(M, N) \otimes_{R} k$ and $\operatorname{Ext}_{R}^{\geqslant t^{\prime}}\left(M^{\prime}, N\right) \otimes_{R} k$ have the same support varieties; thus, $\mathrm{V}(Q, \boldsymbol{f} ; M, N)=\mathrm{V}\left(Q, \boldsymbol{f} ; M^{\prime}, N\right)$.

A similar argument shows that $\mathrm{V}\left(Q, \boldsymbol{f} ; M^{\prime}, N\right)=\mathrm{V}\left(Q, \boldsymbol{f} ; M^{\prime}, N^{\prime}\right)$.

The key result below describes $\mathrm{V}(Q, R ; M, N)$ as the set of unstable directions in the affine space $\widetilde{Q} \mathfrak{a} / \tilde{\mathfrak{n}} \mathfrak{a}$, that is, those directions along which the property that $\operatorname{Ext}^{n}(\widetilde{M}, \widetilde{N})$ vanishes for $n \gg 0$ is lost under passage from $\widetilde{Q}$ to $\widetilde{Q} /(f)$. In the proof of the theorem we adapt an idea from the proof of $[5,(3.9)]$ and use 1.1.4.

2.5. Theorem. Let $Q$ be a local ring with residue field $k$, let $\boldsymbol{f}=f_{1}, \ldots, f_{c}$ be a regular sequence, and let $M, N$ be finite modules over $R=Q /(\boldsymbol{f})$ such that $\operatorname{Ext}_{Q}^{n}(M, N)=0$ for $n \gg 0$. If $Q \subseteq \widetilde{Q}$ is a residual algebraic closure of $Q$, then

$$
\mathrm{V}(Q, \boldsymbol{f} ; M, N)=\left\{\begin{array}{l|l}
\overline{\boldsymbol{a}} \in \widetilde{k}^{c} & \begin{array}{c}
\operatorname{Ext}_{Q_{\boldsymbol{a}}}^{n}(\tilde{M}, \tilde{N}) \neq 0 \\
\text { for infinitely many } n
\end{array}
\end{array}\right\} \cup\{0\}
$$

where $\boldsymbol{a}=\left(a_{1}, \ldots, a_{c}\right) \in \widetilde{Q}^{c}$ denotes some lifting of $\overline{\boldsymbol{a}}=\left(\bar{a}_{1}, \ldots, \bar{a}_{c}\right) \in \widetilde{k}^{c}$ and

$$
Q_{\boldsymbol{a}}=\widetilde{Q} / f_{\boldsymbol{a}} \widetilde{Q} \quad \text { with } \quad f_{\boldsymbol{a}}=a_{1} f_{1}+\cdots+a_{c} f_{c} \in \widetilde{Q} \mathfrak{a}
$$


Proof. By 2.2 we may assume that $Q=\widetilde{Q}$. We use the notation of 1.1 and 2.1. In particular, $\delta=R\left[\chi_{1}, \ldots, \chi_{c}\right]$ is the ring of cohomology operators defined by $\boldsymbol{f}$, and $\mathcal{E}$ is the graded module $\operatorname{Ext}_{R}^{*}(M, N) \otimes_{R} k$ over $\mathcal{R}=$ $s \otimes_{R} k \cong k\left[\chi_{1}, \ldots, \chi_{c}\right]$.

The origin of $k^{c}$ is contained in $\mathrm{V}(Q, f ; M, N)$ by definition, so we may assume that $\bar{a}_{i} \neq 0$ for some $i$. Changing the generating set of the ideal $(\boldsymbol{f})$, we may further assume that $\left(a_{1}, a_{2}, \ldots, a_{c}\right)=(0, \ldots, 0,1)$, hence $f_{\boldsymbol{a}}=f_{c}$.

The image $\boldsymbol{f}^{\prime}$ of the sequence $f_{1}, \ldots, f_{c-1}$ is regular on $Q^{\prime}=Q /\left(f_{c}\right)$. Applying 1.1.1 to the natural projection $Q \rightarrow Q^{\prime}$, the identity maps of $R, M$, $N$, and the $(c-1) \times c$ matrix $\left(q_{i j}\right)$ with $q_{i j}=1$ if $j=i$ and $q_{i j}=0$ otherwise, we see that the algebra of cohomology operators $\delta^{\prime}=R\left[\chi_{1}^{\prime}, \ldots, \chi_{c-1}^{\prime}\right]$ defined by $\boldsymbol{f}^{\prime}$ acts on $\operatorname{Ext}_{R}^{*}(M, N)$ through the embedding $\delta^{\prime} \subseteq \delta$ that maps $\chi_{j}^{\prime}$ to $\chi_{j}$ for $1 \leq j \leq c-1$.

From 1.1.3 and 1.1.4 we see that $\operatorname{Ext}_{Q^{\prime}}^{n}(M, N)=0$ for $n \gg 0$ if and only if the $\delta^{\prime}$-module $\operatorname{Ext}_{R}^{*}(M, N)$ is finite. By Nakayama's Lemma, that happens if and only if $\mathcal{E}$ is finite over $\mathcal{R}^{\prime}={\delta^{\prime}}^{\prime} \otimes_{R} k \cong k\left[\chi_{1}^{\prime}, \ldots, \chi_{c-1}^{\prime}\right]$ and this is equivalent to the finiteness over $\mathcal{R}^{\prime} /\left(\chi_{1}^{\prime}, \ldots, \chi_{c-1}^{\prime}\right) \cong k$ of the module $\mathcal{E} /\left(\chi_{1}^{\prime}, \ldots, \chi_{c-1}^{\prime}\right) \mathcal{E}=\mathscr{E} /\left(\chi_{1}, \ldots, \chi_{c-1}\right) \mathcal{E}$. Denoting this module $\overline{\mathcal{E}}$, observe that $\operatorname{rank}_{k} \overline{\mathscr{E}}$ is finite if and only if $\operatorname{Supp}_{\mathcal{R}}(\overline{\mathscr{E}})=\left\{\left(\chi_{1}, \ldots, \chi_{c}\right)\right\} \subseteq \operatorname{Spec} \mathcal{R}$. As the $\mathcal{R}$-module $\mathcal{E}$ is finite, we get

$$
\operatorname{Supp}_{\mathcal{R}}(\overline{\mathcal{E}})=\operatorname{Supp}_{\mathcal{R}}\left(\mathscr{E} \otimes_{\mathcal{R}} \overline{\mathcal{R}}\right)=\operatorname{Supp}_{\mathcal{R}} \mathscr{E} \cap \operatorname{Supp}_{\mathcal{R}} \overline{\mathcal{R}}
$$

where $\overline{\mathcal{R}}=\mathcal{R} /\left(\chi_{1}, \ldots, \chi_{c-1}\right)$. The Nullstellensatz translates this into

$$
\mathrm{V}(Q, \boldsymbol{f} ; M, N) \cap k(0, \ldots, 0,1)=\{0\} \subseteq k^{c} .
$$

Summing up the discussion, we have: $\operatorname{Ext}_{Q^{\prime}}^{n}(M, N)=0$ for $n \gg 0$ if and only if $k(0, \ldots, 0,1) \nsubseteq \mathbb{\mathrm { V }}(Q, \boldsymbol{f} ; M, N)$. This is another way to state the theorem.

2.6. For each finite $R$-module $M$, varieties $\mathrm{V}(Q, f ; M)$ and $\mathrm{W}(Q, f ; M)$ are defined in $[5$, pp. 82,91$]$ to be the zero-sets in $\widetilde{k}^{c}$ of the annihilators in $\mathcal{R}$ of $\operatorname{Ext}_{R}^{*}(M, k)$ and $\operatorname{Ext}_{R}^{*}(k, M)$, respectively. Thus, the following equalities hold by definition:

$$
\mathrm{V}(Q, \boldsymbol{f} ; M)=\mathrm{V}(Q, \boldsymbol{f} ; M, k) \quad \text { and } \quad \mathrm{W}(Q, \boldsymbol{f} ; M)=\mathrm{V}(Q, \boldsymbol{f} ; k, M) .
$$

The preceding results yield some known properties of these varieties.

2.6.1. If $M^{\prime}$ is a syzygy of $M$ over $R$, then $\mathrm{V}\left(Q, \boldsymbol{f} ; M^{\prime}\right)=\mathrm{V}(Q, \boldsymbol{f} ; M)$. 


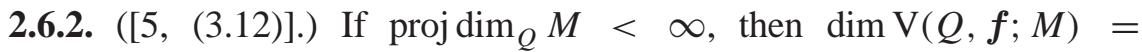
$\mathrm{cx}_{R} M \leq c$.

If inj $\operatorname{dim}_{Q} M<\infty$, then $\operatorname{dim} \mathrm{W}(Q, f ; M)=\mathrm{px}_{R} M \leq c$.

2.6.3. [5, (3.11) and p. 91]. As $\operatorname{Ext}_{Q^{\prime}}^{n}(M, k)=0$ (respectively, $\operatorname{Ext}_{Q^{\prime}}^{n}(k, M)$ $=0$ ) for $n \gg 0$ if and only if proj $\operatorname{dim}_{Q^{\prime}} M$ (respectively, inj $\operatorname{dim}_{Q^{\prime}} M$ ) is finite, we have:

If $\operatorname{proj}_{\operatorname{dim}_{Q}} M<\infty$ then

$$
\mathrm{V}(Q, \boldsymbol{f} ; M)=\left\{\overline{\boldsymbol{a}} \in k^{c} \mid \operatorname{proj} \operatorname{dim}_{Q_{\boldsymbol{a}}} M=\infty\right\} \cup\{0\} .
$$

If inj $\operatorname{dim}_{Q} M<\infty$ then

$$
\mathrm{W}(Q, \boldsymbol{f} ; M)=\left\{\overline{\boldsymbol{a}} \in k^{c} \mid \operatorname{inj} \operatorname{dim}_{Q_{\boldsymbol{a}}} M=\infty\right\} \cup\{0\} .
$$

2.6.4. [5, (5.3)] If $R$ is Gorenstein and $\operatorname{proj}_{\operatorname{dim}} M<\infty$, then $\mathrm{V}(Q, f ; M)$ $=\mathrm{W}(Q, f ; M)$ and $\operatorname{cx}_{R} M=\mathrm{px}_{R} M$. Indeed, in that case $Q$ and $Q_{a}$ are Gorenstein as well, so over them finiteness of projective or injective dimension coincide.

Here is a first link between the variety of a pair and the varieties of its members.

2.7. Corollary. Let $M$ be a finite R-module.

If $\operatorname{proj}_{\operatorname{dim}_{Q}} M<\infty$ then $\mathrm{V}(Q, f ; M, N) \subseteq \mathrm{V}(Q, \boldsymbol{f} ; M)$, socx $\operatorname{cx}_{R}(M, N)$ $\leq \mathrm{cx}_{R} M$.

If inj $\operatorname{dim}_{Q} N<\infty$ then $\mathrm{V}(Q, \boldsymbol{f} ; M, N) \subseteq \mathrm{W}(Q, \boldsymbol{f} ; N)$, so $\operatorname{cx}_{R}(M, N)$ $\leq \mathrm{px}_{R} N$.

Proof. If $\overline{\boldsymbol{a}} \in \mathrm{V}(Q, \boldsymbol{f} ; M, N)$ then $\operatorname{Ext}_{Q_{\boldsymbol{a}}}^{n}(M, N) \neq 0$ for infinitely many $n$, hence both proj $\operatorname{dim}_{Q_{a}} M$ and inj $\operatorname{dim}_{Q_{a}} N$ are infinite. From the theorem we conclude that $\overline{\boldsymbol{a}}$ is in $\mathrm{V}(Q, \boldsymbol{f} ; M)$, respectively, that $\overline{\boldsymbol{a}}$ is in $\mathrm{W}(Q, \boldsymbol{f} ; N)$.

\section{Defining equations}

The definition of cohomology varieties in 2.1 presupposes a knowledge of Ext's as graded modules over a ring of cohomology operators; therefore, it can rarely be used for computations. When $M$ has finite projective dimension over $Q$ we exhibit a determinantal ideal with zero-set $\mathrm{V}(Q, f ; M)$. Rather remarkably, the matrices involved are defined solely in terms of a finite free resolution of $M$ over $Q$. The converse problem - to find a module with prescribed variety - is still open, except for divisors, cf. [5, (6.3)]; the situation in codimension 2 is analyzed in [7]. 
3.1. Let $\boldsymbol{f}=f_{1}, \ldots, f_{c}$ be a regular sequence in a commutative ring $Q$, let $R=Q /(\boldsymbol{f})$, let $M$ be an $R$-module, and let $(\boldsymbol{E}, \partial)$ be a free resolution of $M$ over $Q$.

For $\boldsymbol{j}=\left(j_{1}, \ldots, j_{c}\right) \in \mathbb{N}^{c}$, let $|\boldsymbol{j}|=j_{1}+\cdots+j_{c}$, and for $i=1 \ldots, c$ let $\boldsymbol{e}_{i} \in \mathbb{N}^{c}$ be the $c$-tuple with $\left|\boldsymbol{e}_{i}\right|=1$ and 1 appearing in $i$ 'th position. A system of higher homotopies for $\boldsymbol{f}$ on $\boldsymbol{E}$ is a family of homogeneous $Q$-linear homomorphisms

$$
\boldsymbol{\sigma}=\left\{\sigma^{(\boldsymbol{j})}: \boldsymbol{E} \rightarrow \boldsymbol{E} \mid \sigma_{n}^{(\boldsymbol{j})}: E_{n} \rightarrow E_{n+2|\boldsymbol{j}|-1} \text { for } n \in \mathbb{N} \text { and } \boldsymbol{j} \in \mathbb{N}^{c}\right\}
$$

that satisfy the identities

$$
\begin{gathered}
\sigma^{(\mathbf{0})}=\partial ; \\
\sigma^{(\mathbf{0})} \sigma^{\left(\boldsymbol{e}_{j}\right)}+\sigma^{\left(\boldsymbol{e}_{j}\right)} \sigma^{(\mathbf{0})}=f_{j} \text { id }_{\boldsymbol{E}} \text { for } 1 \leq j \leq c \\
\sum_{\boldsymbol{j}^{\prime}+\boldsymbol{j}^{\prime \prime}=\boldsymbol{j}} \sigma^{\left(\boldsymbol{j}^{\prime}\right)} \sigma^{\left(\boldsymbol{j}^{\prime \prime}\right)}=0 \text { for } \boldsymbol{j} \in \mathbb{N}^{c} \text { with }|\boldsymbol{j}| \geq 2 .
\end{gathered}
$$

The first two conditions imply that for $j=1, \ldots, c$ the degree 1 endomorphism $\sigma^{\left(\boldsymbol{e}_{j}\right)}$ of $\boldsymbol{E}$ is a homotopy between multiplication by $f_{j}$ and the zero map.

Conversely, basic homological algebra yields for $j=1, \ldots, c$ homotopies $\sigma^{\left(e_{j}\right)}$ between $f_{j}$ id $_{\boldsymbol{E}}$ and $0_{\boldsymbol{E}}$. By [16, (8.1)], cf. also [5, (2.4)], such a family of homotopies can be extended to a system $\sigma=\left\{\sigma^{(j)} \mid \boldsymbol{j} \in \mathbb{N}^{c}\right\}$ of higher homotopies for $f$.

After a (tacit) choice of bases, we do not distinguish between a $Q$-linear map of free modules $E_{n}$ and the matrix that represents it.

3.2. Theorem. Let $R=Q /(\boldsymbol{f})$ where $Q$ is a local ring and $\boldsymbol{f}=f_{1}, \ldots, f_{c}$ is a $Q$-regular sequence. Let $M$ be an $R$-module that has a finite free resolution $\boldsymbol{E}$ over $Q$ with $E_{n}=0$ for $n>2 u+1$, set $r=\sum_{i=0}^{u} \operatorname{rank}_{Q} E_{2 i}$, let $\boldsymbol{\sigma}=\left\{\sigma^{(j)} \mid \boldsymbol{j} \in \mathbb{N}^{c}\right\}$ be a system of higher homotopies for $\boldsymbol{f}$ on $\boldsymbol{E}$, and consider the $2 r \times 2 r$ matrix

$$
\sigma(x)=\left(\begin{array}{ccccccc}
0 & \sigma_{1}^{(0)}(x) & 0 & \ldots & 0 & 0 & 0 \\
\sigma_{0}^{(1)}(x) & 0 & \sigma_{2}^{(0)}(x) & \ldots & 0 & 0 & 0 \\
0 & \sigma_{1}^{(1)}(x) & 0 & \ldots & 0 & 0 & 0 \\
\vdots & \vdots & \vdots & \ddots & \vdots & \vdots & \vdots \\
\sigma_{0}^{(u)}(x) & 0 & \sigma_{2}^{(u-1)}(x) & \ldots & 0 & \sigma_{2 u}^{(0)}(x) & 0 \\
0 & \sigma_{1}^{(u)}(x) & 0 & \ldots & \sigma_{2 u-1}^{(1)}(x) & 0 & \sigma_{2 u+1}^{(0)}(x) \\
\sigma_{0}^{(u+1)}(x) & 0 & \sigma_{2}^{(u)}(x) & \ldots & 0 & \sigma_{2 u}^{(1)}(x) & 0
\end{array}\right)
$$


with elements in the polynomial ring $Q[x]=Q\left[x_{1}, \ldots, x_{c}\right]$, where

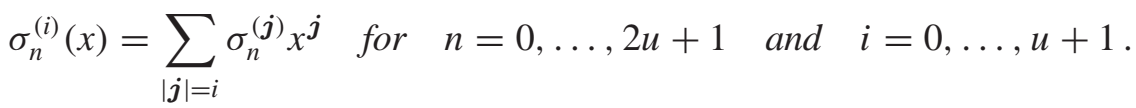

The algebraic set $\mathrm{V}(Q, f ; M)$ is then defined by the vanishing of the $r \times r$ minors of the matrix $\bar{\sigma}(x)$ obtained by reducing $\sigma(x)$ modulo the maximal ideal of $Q$.

Proof. By 2.2 we may assume that $k$ is algebraically closed.

The complex $\boldsymbol{E}[x]=\boldsymbol{E} \otimes_{Q} Q[x]$ is a free resolution of $M[x]=$ $M \otimes_{Q} Q[x]$, and

$$
\boldsymbol{\sigma}(x)=\left\{\sigma^{(i)}(x)=\sum_{|\boldsymbol{j}|=i} \sigma^{(\boldsymbol{j})} x^{\boldsymbol{j}}: \boldsymbol{E}[x] \rightarrow \boldsymbol{E}[x] \mid i \in \mathbb{N}\right\}
$$

is a family of $Q[x]$-linear homomorphisms. Setting $f=f_{1} x_{1}+\cdots+f_{c} x_{c}$, $\dot{Q}=Q[x] / f Q[x]$, and $\dot{E}_{n}=E_{n}[x] \otimes_{Q[x]} \dot{Q}$, denote $\boldsymbol{G}$ the graded $\dot{Q}$ module $G$ with

$$
G_{2 i}=\dot{E}_{0} \oplus \dot{E}_{2} \oplus \cdots \oplus \dot{E}_{2 i} \quad \text { and } \quad G_{2 i+1}=\dot{E}_{1} \oplus \dot{E}_{3} \oplus \cdots \oplus \dot{E}_{2 i+1}
$$

for $i \in \mathbb{Z}$. The maps $\dot{\sigma}_{n}^{(i)}=\sigma_{n}^{(i)}(x) \otimes_{Q[x]} \dot{Q}$ yield a homomorphism $\partial: \boldsymbol{G} \rightarrow$ $G$ with

$$
\begin{aligned}
& \partial_{2 i+1}=\left(\begin{array}{cccccc}
\dot{\sigma}_{1}^{(0)} & 0 & 0 & \ldots & 0 & 0 \\
\dot{\sigma}_{1}^{(1)} & \dot{\sigma}_{3}^{(0)} & 0 & \ldots & 0 & 0 \\
\dot{\sigma}_{1}^{(2)} & \dot{\sigma}_{3}^{(1)} & \dot{\sigma}_{5}^{(0)} & \ldots & 0 & 0 \\
\vdots & \vdots & \vdots & \ddots & \vdots & \vdots \\
\dot{\sigma}_{1}^{(i-2)} & \dot{\sigma}_{3}^{(i-3)} & \dot{\sigma}_{5}^{(i-4)} & \ldots & \dot{\sigma}_{2 i-1}^{(0)} & 0 \\
\dot{\sigma}_{1}^{(i-1)} & \dot{\sigma}_{3}^{(i-2)} & \dot{\sigma}_{5}^{(i-3)} & \ldots & \dot{\sigma}_{2 i-1}^{(1)} & \dot{\sigma}_{2 i+1}^{(0)}
\end{array}\right): G_{2 i+1} \rightarrow G_{2 i}, \\
& \partial_{2 i+2}=\left(\begin{array}{ccccccc}
\dot{\sigma}_{0}^{(1)} & \dot{\sigma}_{2}^{(0)} & 0 & \ldots & 0 & 0 & 0 \\
\dot{\sigma}_{0}^{(2)} & \dot{\sigma}_{2}^{(1)} & \dot{\sigma}_{4}^{(0)} & \ldots & 0 & 0 & 0 \\
\dot{\sigma}_{0}^{(3)} & \dot{\sigma}_{2}^{(2)} & \dot{\sigma}_{4}^{(1)} & \ldots & 0 & 0 & 0 \\
\vdots & \vdots & \vdots & \ddots & \vdots & \vdots & \\
\dot{\sigma}_{0}^{(i)} & \dot{\sigma}_{2}^{(i-1)} & \dot{\sigma}_{4}^{(i-2)} & \ldots & \dot{\sigma}_{2 i-2}^{(1)} & \dot{\sigma}_{2 i}^{(0)} & 0 \\
\dot{\sigma}_{0}^{(i+1)} & \dot{\sigma}_{2}^{(i)} & \dot{\sigma}_{4}^{(i-1)} & \ldots & \dot{\sigma}_{2 i-2}^{(2)} & \dot{\sigma}_{2 i}^{(1)} & \dot{\sigma}_{2 i+2}^{(0)}
\end{array}\right): G_{2 i+2} \rightarrow G_{2 i+1} .
\end{aligned}
$$

Simple calculations show that $\boldsymbol{\sigma}(x)$ is a system of higher homotopies for $f$ on $\boldsymbol{E}[x]$. 
In particular, $(\boldsymbol{G}, \partial)$ is a complex. The assumption on $E_{n}$ implies that $\sigma_{n}^{(i)}=0$ for $n>2(u-i+1)$, so $G_{n+2}=G_{n}$ and $\partial_{n+3}=\partial_{n+1}$ for $n \geq 2 u$, hence $\boldsymbol{G}_{\geqslant 2 u}$ is isomorphic to the complex

$$
C=\cdots \longrightarrow C \stackrel{\gamma}{\longrightarrow} D \stackrel{\delta}{\longrightarrow} C \stackrel{\gamma}{\longrightarrow} D \stackrel{\delta}{\longrightarrow} C \longrightarrow 0
$$

where

$$
C=G_{2 u}, \quad D=G_{2 u+1}, \quad \delta=\partial_{2 u+1}, \quad \gamma=\partial_{2 u+2} .
$$

We have $\operatorname{rank}_{Q} C=\operatorname{rank}_{Q} D=r$ : since $f_{1} M=0$ and $f_{1}$ is $Q$-regular the Euler characteristic of $\boldsymbol{E}$ vanishes, so $\sum_{i=0}^{u} r_{2 i+1}=\sum_{i=0}^{u} r_{2 i}=r$ for $r_{n}=\operatorname{rank}_{Q} E_{n}$.

For each $\boldsymbol{a}=\left(a_{1}, \ldots, a_{c}\right) \in Q^{c}$ there are obvious isomorphisms of rings

$$
\frac{Q[x]}{\left(x_{1}-a_{1}, \ldots, x_{c}-a_{c}\right) Q[x]} \cong Q \text { and } \frac{\dot{Q}}{\left(x_{1}-a_{1}, \ldots, x_{c}-a_{c}\right) \dot{Q}} \cong Q_{a} .
$$

Using this $Q[x]$-module structure on $Q$ we obtain a family of higher homotopies $\boldsymbol{\sigma} \otimes_{Q[x]} Q$ for $f_{\boldsymbol{a}}=a_{1} f_{1}+\cdots a_{c} f_{c}$ on $\boldsymbol{E}[x] \otimes_{Q[x]} Q=\boldsymbol{E}$.

If $\overline{\boldsymbol{a}} \neq 0$ then $f_{\boldsymbol{a}}$ is a non-zero-divisor on $Q$, so $\boldsymbol{G}_{\boldsymbol{a}}=\boldsymbol{G} \otimes_{\dot{Q}} Q_{\boldsymbol{a}}$ is a free resolution of $M$ over $Q_{a}$ by a theorem of Shamash [31, §3], cf. also [6, (3.1.3)]. Setting $C_{\boldsymbol{a}}=C \otimes_{\dot{Q}} Q_{a}, D_{\boldsymbol{a}}=D \otimes_{\dot{Q}} Q_{\boldsymbol{a}}$, and denoting $\delta_{\boldsymbol{a}}, \gamma_{\boldsymbol{a}}$ the evaluations at $\boldsymbol{a}$ of the polynomial matrices $\delta, \gamma$, we obtain the tail of that resolution in the form

$$
C_{a}=\cdots \longrightarrow C_{a} \stackrel{\gamma_{a}}{\longrightarrow} D_{a} \stackrel{\delta_{a}}{\longrightarrow} C_{a} \stackrel{\gamma_{a}}{\longrightarrow} D_{a} \stackrel{\delta_{a}}{\longrightarrow} C_{a} \longrightarrow 0 .
$$

Now 2.6.3 shows that $\overline{\boldsymbol{a}} \in \mathrm{V}(Q, \boldsymbol{f} ; M)$ if and only if $\operatorname{proj}_{\operatorname{dim}_{Q_{a}}} M=\infty$. This happens if and only if the following complex of $k$-vector spaces is not exact

$$
\bar{C}_{a}=\cdots \longrightarrow \bar{C}_{a} \stackrel{\bar{\gamma}_{a}}{\longrightarrow} \bar{D}_{a} \stackrel{\bar{\delta}_{a}}{\longrightarrow} \bar{C}_{a} \stackrel{\bar{\gamma}_{a}}{\longrightarrow} \bar{D}_{a} \stackrel{\bar{\delta}_{a}}{\longrightarrow} \bar{C}_{a} \longrightarrow \cdots
$$

Non-exactness of $\overline{\boldsymbol{C}}_{\boldsymbol{a}}$ is equivalent to non-exactness of the sequence

$$
\bar{C}_{a} \oplus \bar{D}_{a} \stackrel{\beta_{a}}{\longrightarrow} \bar{C}_{a} \oplus \bar{D}_{a} \stackrel{\beta_{a}}{\longrightarrow} \bar{C}_{a} \oplus \bar{D}_{a} \text { where } \beta_{a}=\left(\begin{array}{cc}
0 & \bar{\gamma}_{a} \\
\bar{\delta}_{a} & 0
\end{array}\right) \text {. }
$$

Since $\beta_{\boldsymbol{a}}^{2}=0$, the latter property translates into $\operatorname{rank}_{k} \beta_{\boldsymbol{a}}<\operatorname{rank}_{k} \operatorname{Ker} \beta_{\boldsymbol{a}}$; as $\operatorname{rank}_{k} \operatorname{Ker} \beta_{\boldsymbol{a}}=2 r-\operatorname{rank}_{k} \beta_{\boldsymbol{a}}$ this means that $\operatorname{rank}_{k} \beta_{\boldsymbol{a}}<r$. The isomorphisms

$$
\bar{C}_{\boldsymbol{a}} \oplus \bar{D}_{\boldsymbol{a}} \cong \bigoplus_{i=0}^{u} \bar{E}_{2 i} \oplus \bigoplus_{i=0}^{u} \bar{E}_{2 i+1} \cong \bigoplus_{i=0}^{2 u+1} \bar{E}_{i}
$$

take $\beta_{\boldsymbol{a}}$ to $\bar{\sigma}(\overline{\boldsymbol{a}})$, so the latest inequality reads $\operatorname{rank}_{k} \bar{\sigma}(\overline{\boldsymbol{a}})<r$.

Letting $\boldsymbol{a}$ range over $Q^{c} \backslash \mathfrak{m} Q^{c}$, we obtain the desired description of $\mathrm{V}(Q, f ; M)$ as the zero-set of the ideal of $r \times r$ minors of the matrix $\bar{\sigma}(x)$. 
Recall that the grade of $M$ is the length of a maximal $Q$-sequence contained in the annihilator $\operatorname{ann}_{Q} M$; it is denoted grade ${ }_{Q} M$ and satisfies grade $_{Q} M \leq \operatorname{proj~dim}_{Q} M$. If equality holds and $M$ is finite over $Q$, then $M$ is said to be a perfect $Q$-module.

3.3. Theorem. Let $Q$ be a local ring, $f$ a $Q$-regular sequence, and $R=$ $Q /(\boldsymbol{f})$. If an $R$-module $M$ is perfect of grade $g$ over $Q$, then so is $M^{\dagger}=$ $\operatorname{Ext}_{R}^{g-c}(M, R)$, and

$$
\mathrm{V}\left(Q, \boldsymbol{f} ; M^{\dagger}\right)=\mathrm{V}(Q, \boldsymbol{f} ; M) .
$$

Proof. By a classical isomorphism of Rees, $\operatorname{Ext}_{R}^{g-c}(M, R) \cong \operatorname{Ext}_{Q}^{g}(M, Q)$.

Let $\boldsymbol{E}$ be a finite free resolution of $M$ over $Q$ with $E_{n}=0$ for $n>g$. As a $Q$-regular sequence of length $g$ annihilates $M$, the complex $\operatorname{Hom}_{Q}(\boldsymbol{E}, Q)$ has cohomology only in degree $g$, where it is isomorphic to $M^{\dagger}$. Setting $E_{n}^{*}=\operatorname{Hom}_{Q}\left(E_{g-n}, Q\right)$ and $\partial_{n}^{*}=\operatorname{Hom}_{Q}\left(\partial_{g-n+1}, Q\right)$, we get for $M^{\dagger} \mathrm{a}$ $Q$-free resolution

$$
\begin{aligned}
\boldsymbol{E}^{*}=0 & \longrightarrow E_{g}^{*} \longrightarrow \ldots \longrightarrow E_{n}^{*} \stackrel{\partial_{n}^{*}}{\longrightarrow} E_{n-1}^{*} \longrightarrow \ldots \\
& \longrightarrow E_{0}^{*} \longrightarrow 0 .
\end{aligned}
$$

Choose a system $\boldsymbol{\sigma}$ of higher homotopies for $\boldsymbol{f}$ on $\boldsymbol{E}$ and set

$$
\left(\sigma_{n}^{(\boldsymbol{j})}\right)^{*}=\operatorname{Hom}_{Q}\left(\sigma_{g-n-2|\boldsymbol{j}|+1}^{(\boldsymbol{j})}, Q\right): E_{n}^{*} \rightarrow E_{n+2|\boldsymbol{j}|-1}^{*}
$$

for $n \in \mathbb{Z}$ and $j \in \mathbb{N}^{c}$. Dualizing the identities satisfied by $\sigma$, we see that $\boldsymbol{\sigma}^{*}=\left\{\sigma^{(\boldsymbol{j})}: \boldsymbol{E}^{*} \rightarrow \boldsymbol{E}^{*} \mid\left(\sigma_{n}^{(\boldsymbol{j})}\right)^{*}: E_{n}^{*} \rightarrow E_{n+2|\boldsymbol{j}|-1}^{*}\right.$ for $n \in \mathbb{N}$ and $\left.\boldsymbol{j} \in \mathbb{N}^{c}\right\}$

is a system of higher homotopies for $\boldsymbol{f}$ on $\boldsymbol{E}^{*}$. Fixing an integer $u$ with $2 u+1 \geq g$, form the matrix $\boldsymbol{\sigma}^{*}(x)$ as defined in Theorem 3.2. Writing $\iota_{t}$ for the $t \times t$ unit matrix, we see by a direct computation that $\sigma^{*}(x)=\omega \sigma(x)^{\top} \omega$, where

$$
\omega=\left(\begin{array}{ccccccc}
0 & 0 & 0 & \ldots & 0 & 0 & \iota_{r_{2 u+1}} \\
0 & 0 & 0 & \ldots & 0 & \iota_{r_{2 u}} & 0 \\
0 & 0 & 0 & \ldots & \iota_{r_{2 u-1}} & 0 & 0 \\
\vdots & \vdots & \vdots & \ddots & \vdots & \vdots & \vdots \\
0 & 0 & \iota_{r_{2}} & \ldots & 0 & 0 & 0 \\
0 & \iota_{r_{1}} & 0 & \ldots & 0 & 0 & 0 \\
\iota_{r_{0}} & 0 & 0 & \ldots & 0 & 0 & 0
\end{array}\right)
$$

and $\sigma(x)^{\top}$ is the transpose of $\sigma(x)$. Thus, the ideals of $r \times r$ minors of the matrices $\boldsymbol{\sigma}^{*}(x)$ and $\boldsymbol{\sigma}(x)$ coincide, hence $\mathrm{V}\left(Q, \boldsymbol{f} ; M^{\dagger}\right)=\mathrm{V}(Q, \boldsymbol{f} ; M)$ by Theorem 3.2. 


\section{Finite CI-dimension}

The theme of this section is that in certain cases, a 'sufficiently big' gap in a (co)homology sequence forces the entire sequence to vanish after a point that can be predicted accurately. We consider a class of modules, introduced in [8], that includes the modules of finite projective dimension over any ring, cf. 4.5.1, and all modules over 1.c.i. rings, cf. 6.2, but is strictly larger, as shown in $[8,(3.2)]$.

A quasi-deformation (of codimension $c$ ) of a local ring $R$ is a diagram of local homomorphisms $R \rightarrow R^{\prime} \leftarrow Q$, the first being faithfully flat and the second surjective with kernel generated by a $Q$-regular sequence (of length $c$ ).

Let $M \neq 0$ be a finite module over a noetherian ring $R$. If $R$ is local, set

CI- $\operatorname{dim}_{R} M=$

$$
\inf \left\{\begin{array}{l|l}
\operatorname{proj}_{\operatorname{dim}_{Q}}\left(M \otimes_{R} R^{\prime}\right)-\operatorname{proj} \operatorname{dim}_{Q} R^{\prime} & \begin{array}{l}
R \rightarrow R^{\prime} \leftarrow Q \text { is a } \\
\text { quasi-deformation }
\end{array}
\end{array}\right\} ;
$$

in general, the complete intersection (or, CI-)dimension of $M$ over $R$ is defined by

CI- $\operatorname{dim}_{R} M=\sup \left\{\mathrm{CI}-\operatorname{dim}_{R_{\mathfrak{m}}} M_{\mathfrak{m}} \mid \mathfrak{m} \in \operatorname{Max}(R)\right\} \quad$ and $\quad$ CI- $\operatorname{dim}_{R} 0=0$.

Many arguments involving finite CI-dimension hinge upon the following results.

4.1. Let $R$ be a local ring, let $M$ be a finite $R$-module with CI- $\operatorname{dim}_{R} M<\infty$, and let $R \rightarrow R^{\prime} \leftarrow Q$ is a quasi-deformation of codimension $c$ such that the module $M^{\prime}=M \otimes_{R} R^{\prime}$ has finite projective dimension over $Q$.

4.1.1. For each $R$-module $N$ there are isomorphisms of $R^{\prime}$-modules

$$
\operatorname{Ext}_{R}^{n}(M, N) \otimes_{R} R^{\prime} \cong \operatorname{Ext}_{R^{\prime}}^{n}\left(M^{\prime}, N \otimes_{R} R^{\prime}\right) \quad \text { for } \quad n \in \mathbb{Z}
$$

and thus $v_{R}\left(\operatorname{Ext}_{R}^{n}(M, N)\right)=v_{R^{\prime}}\left(\operatorname{Ext}_{R^{\prime}}^{n}\left(M^{\prime}, N \otimes_{R} R^{\prime}\right)\right)$.

4.1.2. If $N$ is a finite $R$-module, then Proposition 1.3 and Corollary 2.7 yield

$$
\operatorname{cx}_{R}(M, N)=\operatorname{cx}_{R^{\prime}}\left(M^{\prime}, N \otimes_{R} R^{\prime}\right) \leq \operatorname{cx}_{R^{\prime}} M^{\prime}=\operatorname{cx}_{R} M \leq c .
$$

4.1.3. There is a quasi-deformation $R \rightarrow R^{\prime} \leftarrow Q$ of codimension $\mathrm{cx}_{R} M$, with proj $\operatorname{dim}_{Q} M^{\prime}<\infty$, and ring $Q$ with algebraically closed residue field, cf. [8, (5.10)]. 
4.1.4. Let $\boldsymbol{F}$ be a minimal free resolution of $M$; the quasi-deformation above can be chosen so that the complex $\boldsymbol{F}^{\prime}=\boldsymbol{F} \otimes_{R} R^{\prime}$ admits a chain map $\vartheta: \boldsymbol{F}^{\prime} \rightarrow \boldsymbol{F}^{\prime}$ of degree -2 with $\vartheta\left(F_{n+2}^{\prime}\right)=F_{n}^{\prime}$ for some integer $s \geq 0$ and all $n>s$, cf. [8, (7.2.2)].

4.1.5. The Auslander-Buchsbaum Equality is extended in $[8,(1.4)]$ to:

$$
\text { CI- } \operatorname{dim}_{R} M=\operatorname{depth} R-\operatorname{depth} M \text {. }
$$

First we establish a self-test for finite projective dimension. It vastly generalizes a result of Auslander, Ding, and Solberg [4, (1.8)], which is the case $i=1$.

4.2. Theorem. A finite module $M$ of finite CI-dimension over a noetherian ring $R$ has finite projective dimension if (and only if) $\operatorname{Ext}_{R}^{2 i}(M, M)=0$ for some $i \geq 1$.

Proof. It suffices to prove that proj $\operatorname{dim}_{R_{\mathfrak{m}}} M_{\mathfrak{m}} \leq$ CI-dim $R_{\mathfrak{m}} M_{\mathfrak{m}}$ for each $\mathfrak{m} \in \operatorname{Max}(R)$, so we may assume that $R$ is local with maximal ideal $\mathfrak{m}$.

Let $\boldsymbol{F}$ be a minimal free resolution of $M$, and choose by 4.1 .4 a quasideformation $R \rightarrow R^{\prime} \leftarrow Q$ and a chain map $\vartheta: \boldsymbol{F}^{\prime} \rightarrow \boldsymbol{F}^{\prime}$ of degree -2 on $\boldsymbol{F}^{\prime}=\boldsymbol{F} \otimes_{R} R^{\prime}$ such that $\vartheta\left(F_{n+2}^{\prime}\right)=F_{n}^{\prime}$ for for some integer $s \geq 0$ and all $n>s$. Since

$$
\begin{aligned}
\mathrm{H}_{-2 i} \operatorname{Hom}_{R^{\prime}}\left(\boldsymbol{F}^{\prime}, \boldsymbol{F}^{\prime}\right) & \cong \mathrm{H}_{-2 i} \operatorname{Hom}_{R}(\boldsymbol{F}, \boldsymbol{F}) \otimes_{R} R^{\prime} \\
& \cong \operatorname{Ext}_{R}^{2 i}(M, M) \otimes_{R} R^{\prime}=0
\end{aligned}
$$

the $i$ 'th iterate $\vartheta^{i}: \boldsymbol{F}^{\prime} \rightarrow \boldsymbol{F}^{\prime}$ is null-homotopic, so there is a homomorphism of graded modules $\lambda: \boldsymbol{F}^{\prime} \rightarrow \boldsymbol{F}^{\prime}$ of degree $(-2 i+1)$ with $\vartheta^{i}=\partial \lambda+\lambda \partial$. If $n>s$, then

$$
\begin{aligned}
F_{n}^{\prime}=\vartheta^{i}\left(F_{n+2 i}^{\prime}\right) & \subseteq \partial \lambda\left(F_{n+2 i}^{\prime}\right)+\lambda \partial\left(F_{n+2 i}^{\prime}\right) \\
& \subseteq \partial\left(F_{n+1}^{\prime}\right)+\lambda\left(\mathfrak{m} F_{n+2 i-1}^{\prime}\right) \subseteq \mathfrak{m} F_{n}^{\prime}
\end{aligned}
$$

so $F_{n}^{\prime}=F_{n} \otimes_{R} R^{\prime}$ vanishes by Nakayama's Lemma, and hence $F_{n}=0$. Thus, $M$ has finite projective dimension, so $\operatorname{proj}^{\operatorname{dim}_{R}} M=\mathrm{CI}-\operatorname{dim}_{R} M$ by 4.1.5.

In Theorem 4.2 the vanishing condition cannot be moved, in any complexity, from even to odd Ext's, and the conclusion fails if Ext's are replaced by Tor's. 
4.3. Example. Let $Q=k\left[\left[u_{1}, \ldots, u_{c}, x_{1}, \ldots, x_{c}\right]\right]$ be a ring of formal power series in $2 c$ indeterminates over a field $k$, fix an integer $b$ with $0 \leq b \leq c$, and set

$$
R=\frac{Q}{\left(u_{1} x_{1}, \ldots, u_{c} x_{c}\right)} \quad \text { and } \quad S=\frac{Q}{\left(u_{1}, \ldots, u_{b}, x_{b+1} u_{b+1}, \ldots, x_{c} u_{c}\right)} .
$$

For $b=c=1$ a free resolution of $S \cong k\left[\left[x_{1}\right]\right]$ over $R$ is given by

$$
\boldsymbol{F}=\ldots \stackrel{u_{1}}{\longrightarrow} R \stackrel{x_{1}}{\longrightarrow} R \stackrel{u_{1}}{\longrightarrow} R \stackrel{x_{1}}{\longrightarrow} R \stackrel{u_{1}}{\longrightarrow} R \longrightarrow 0 .
$$

For $i \geq 1$ the homology of the complexes $\operatorname{Hom}_{R}(\boldsymbol{F}, S)$ and $\boldsymbol{F} \otimes_{R} S$ yields $\operatorname{Ext}_{R}^{2 i-1}(S, S) \cong \operatorname{Tor}_{2 i}^{R}(S, S)=0$ and $\operatorname{Ext}_{R}^{2 i}(S, S) \cong \operatorname{Tor}_{2 i-1}^{R}(S, S) \cong k$.

In general, Proposition 1.2 shows that $\operatorname{Ext}_{R}^{*}(S, S)$ is the homology of the Koszul complex on $\left\{x_{1} \chi_{1}, \ldots, x_{b} \chi_{b}, \chi_{b+1}, \ldots, \chi_{c}\right\} \subseteq S\left[\chi_{1} \ldots, \chi_{c}\right]$, and $\operatorname{Ext}_{R}^{*}(S, k)$ is the homology of the one on $\left\{0, \ldots, 0, \chi_{b+1}, \ldots, \chi_{c}\right\} \subseteq$ $k\left[\chi_{1}, \ldots, \chi_{c}\right]$, hence

$$
\begin{gathered}
\operatorname{Ext}_{R}^{*}(S, S) \cong \frac{S\left[\chi_{1}, \ldots, \chi_{b}\right]}{\left(x_{1} \chi_{1}, \ldots, x_{b} \chi_{b}\right)} ; \\
\operatorname{Ext}_{R}^{*}(S, k) \cong k\left[\chi_{1}, \ldots, \chi_{b}\right] \otimes_{k} \bigwedge_{k}^{*}\left(k^{c-b}\right) .
\end{gathered}
$$

These isomorphisms yield, respectively, $\operatorname{Ext}_{R}^{\text {odd }}(S, S)=0$ and $\mathrm{cx}_{R} S=b$.

We now turn to vanishing results that are most succinctly stated in terms of stable (co)homology for modules of finite G-dimension. We review the basics.

4.4. Let $M$ be a finite module over a noetherian ring $R$.

4.4.1. A complete resolution of an $R$-module $M$ is a complex $\boldsymbol{T}$ of finite projective $R$-modules, such that $\mathrm{H}_{n}(\boldsymbol{T})=0=\mathrm{H}^{n}\left(\boldsymbol{T}^{*}\right)$ for all $n \in \mathbb{Z}$, and $\boldsymbol{T}_{\geqslant r}=\boldsymbol{F}_{\geqslant r}$ for some projective resolution $\boldsymbol{F}$ of $M$ and some integer $r$. It is well known that if $M$ has complete resolutions, then any two of them are homotopy equivalent, cf. e.g. [13], or [15, (2.4)] for a published treatment. In particular, the modules

$$
\widehat{\operatorname{Ext}}_{R}^{n}(M, N)=\mathrm{H}^{n} \operatorname{Hom}_{R}(\boldsymbol{T}, N) \text { and } \widehat{\operatorname{Tor}}_{n}^{R}(M, N)=\mathrm{H}_{n}\left(\boldsymbol{T} \otimes_{R} N\right)
$$

are well defined; we refer to them as stable (co)homology modules.

4.4.2. If $\boldsymbol{T}$ is a complete resolution of $M$, then clearly for each $i \in \mathbb{Z}$ the shifted complex $\boldsymbol{T}[-i]$ is a complete resolution of $\Omega^{i} \boldsymbol{T}=\operatorname{Coker}\left(\partial_{i+1}^{\boldsymbol{T}}: T_{i+1}\right.$ $\rightarrow T_{i}$ ), hence

$\widehat{\operatorname{Ext}}_{R}^{n}(M, N)=\widehat{\operatorname{Ext}}_{R}^{n+i}\left(\Omega^{i} \boldsymbol{T}, N\right)$ and $\widehat{\operatorname{Tor}}_{n}^{R}(M, N) \cong \widehat{\operatorname{Tor}}_{n-i}^{R}\left(\Omega^{i} \boldsymbol{T}, N\right)$. 
4.4.3. If $M \neq 0$, then its $G$-dimension is the shortest length of a resolution of $M$ by modules $G$ with $G \cong G^{* *}$ and $\operatorname{Ext}_{R}^{n}(G, R)=0=\operatorname{Ext}_{R}^{n}\left(G^{*}, R\right)$ for all $n>0$; it is denoted G- $\operatorname{dim}_{R} M$.

By a basic result of Auslander and Bridger [3, (4.20)] and Goto [19], a noetherian ring $R$ is Gorenstein if and only if each finite module $M$ has G-dim ${ }_{R} M<\infty$.

4.4.4. The module $M$ has a complete resolution if and only if $\mathrm{G}-\operatorname{dim}_{R} M<\infty$.

If $\operatorname{G-dim}_{R} M=r<\infty$, then choose a resolution $\boldsymbol{F}$ of $M$ by finite projective $R$-modules, set $G=\operatorname{Coker}\left(\partial_{r+1}^{\boldsymbol{F}}: F_{r+1} \rightarrow F_{r}\right)$, and let $\boldsymbol{G}$ be a resolution of $G^{*}$ by finite projective $R$-modules with augmentation $\gamma$. Define a complex $\boldsymbol{T}$ through

$$
T_{n}=\left\{\begin{array}{ll}
F_{n} & \text { for } n \geq r ; \\
G_{r-1-n}^{*} & \text { for } n<r ;
\end{array} \quad \partial_{n}^{\boldsymbol{T}}= \begin{cases}\partial_{n}^{\boldsymbol{F}} & \text { for } n>r \\
\gamma^{*} \beta \alpha & \text { for } n=r \\
\left(\partial_{r-n} \boldsymbol{G}\right)^{*} & \text { for } n<r\end{cases}\right.
$$

where $\alpha: F_{r} \rightarrow G$ and $\beta: G \rightarrow G^{* *}$ are the canonical maps. Clearly, $\boldsymbol{T}$ is a complex of finite projective $R$-modules. The exact sequence of complexes $0 \rightarrow \boldsymbol{G}^{*}[r-1] \rightarrow \boldsymbol{T} \rightarrow \boldsymbol{F}_{\geqslant r} \rightarrow 0$, shows that $\mathrm{H}_{n}(\boldsymbol{T})=0$ for $n>r$, yields an exact sequence

$$
0 \longrightarrow \mathrm{H}_{r}(\boldsymbol{T}) \longrightarrow G \stackrel{\beta}{\longrightarrow} G^{* *} \longrightarrow \mathrm{H}_{r-1}(\boldsymbol{T}) \longrightarrow 0
$$

and isomorphisms $\mathrm{H}_{n}(\boldsymbol{T}) \cong \operatorname{Ext}_{R}^{r-1-n}(G, R)$ for $n<r-1$.

By $[3,(3.15)]$ the module $G$ has G-dimension 0 , so $\beta$ is an isomorphism and $\operatorname{Ext}_{R}^{i}(G, R)=0$ for $i>0$, hence $\mathrm{H}_{n}(\boldsymbol{T})=0$ for all $n \in \mathbb{Z}$. The dualized exact sequence of complexes now shows that $\mathrm{H}_{n}\left(\boldsymbol{T}^{*}\right)$ is isomorphic to $\mathrm{H}_{n}(G[1-r])=0$ for $n>1-r$, to $\operatorname{Ker}\left(\beta^{*}\right)$ for $n=1-r$, to $\operatorname{Coker}\left(\beta^{*}\right)$ for $n=-r$, and to $\mathrm{H}_{n}\left(\left(\boldsymbol{F}_{\geqslant r}\right)^{*}\right)=\operatorname{Ext}_{R}^{n-r}\left(G^{*}, R\right)=0$ for $n<-r$. Thus, $\mathrm{H}_{n}\left(\boldsymbol{T}^{*}\right)=0$ for all $n \in \mathbb{Z}$.

Conversely, if $M$ has a complete resolution, then $\mathrm{G}-\operatorname{dim}_{R} M<\infty$ due to:

4.4.5. If $\boldsymbol{T}$ is a complete resolution, then G- $\operatorname{dim}_{R} \Omega^{i} \boldsymbol{T}=0$ for each $i \in \mathbb{Z}$.

It clearly suffices to deal with $G=\Omega^{0} \boldsymbol{T}$. Since $\boldsymbol{T}_{\geqslant 0}$ is a projective resolution of $G$, applying $\operatorname{Hom}_{R}(, R)$ to the exact sequence of complexes $0 \rightarrow \boldsymbol{T}_{<0} \rightarrow \boldsymbol{T} \rightarrow \boldsymbol{T}_{\geqslant 0} \rightarrow 0$ yields $\operatorname{Ext}_{R}^{n}(G, R)=0$ for $n>0$, and exhibits $\left(\boldsymbol{T}_{<0}\right)^{*}[-1]$ as a projective resolution of $G^{*}$. Dualizing once again we recover the original sequence, which shows that $\operatorname{Ext}_{R}^{n}\left(G^{*}, R\right)=0$ for $n>0$ and that the biduality map $G \rightarrow G^{* *}$ is bijective.

4.4.6. The construction in 4.4 .4 shows that for $n>r$ there are isomorphisms

$$
\widehat{\operatorname{Ext}}_{R}^{n}(M, N) \cong \operatorname{Ext}_{R}^{n}(M, N) \quad \text { and } \quad \widehat{\operatorname{Tor}}_{n}^{R}(M, N) \cong \operatorname{Tor}_{n}^{R}(M, N) \text {. }
$$


4.4.7. Assume that $\mathrm{G}-\operatorname{dim}_{R} M=0$. If $\boldsymbol{T}$ is the complete resolution from 4.4.4, then $\Omega^{0} \boldsymbol{T}=M$ and $\boldsymbol{T}^{*}[-1]$ is a complete resolution of $M^{*}$, hence

$$
\widehat{\operatorname{Tor}}_{n}^{R}(M, N) \cong \widehat{\operatorname{Ext}}_{R}^{-n-1}\left(M^{*}, N\right)
$$

for all $n \in \mathbb{Z}$, due to the canonical isomorphism $T \otimes_{R} N \cong \operatorname{Hom}_{R}\left(\boldsymbol{T}^{*}, N\right)$.

CI-dimension is compared to other homological dimensions in [8, (1.4)].

4.5. Let $M$ be a finite module over a noetherian ring $R$.

4.5.1. There are inequalities $\mathrm{G}-\operatorname{dim}_{R} M \leq \mathrm{CI}-\operatorname{dim}_{R} M \leq \operatorname{proj} \operatorname{dim}_{R} M$, and equalities hold to the left of any finite dimension, cf. [8, (1.4)].

4.5.2. If $0 \rightarrow M^{\prime} \rightarrow F_{n-1} \rightarrow \cdots \rightarrow F_{0} \rightarrow M \rightarrow 0$ is an exact sequence of finite projective $R$-modules $F_{i}$, then CI-dim ${ }_{R} M^{\prime}=\max \left\{\mathrm{CI}-\operatorname{dim}_{R} M-n, 0\right\}$ by $[8,(1.9)]$.

4.6. Proposition. Any finite module $M$ of finite CI-dimension has a complete resolution. Each complete resolution $\boldsymbol{T}$ of $M$ has $\mathrm{CI}-\operatorname{dim}_{R}\left(\Omega^{n} \boldsymbol{T}\right)=0$ for all $n \in \mathbb{Z}$.

Proof. The first assertion follows from 4.5.1 and 4.4.4. For the second it suffices to note that $M$ and $\Omega^{n} \boldsymbol{T}$ have a common syzygy, and apply 4.5.2.

To any finite $R$-module $M$ over a noetherian $\operatorname{ring} R$ we attach a number

$$
\operatorname{cx}_{R} M=\sup \left\{\operatorname{cx}_{R_{\mathfrak{m}}} M_{\mathfrak{m}} \mid \mathfrak{m} \in \operatorname{Max}(R) \cap \operatorname{Supp}(M)\right\} .
$$

In conditions (i) or (iv) of our next vanishing result on cohomology, the number of consecutive Ext's may not be decreased in general, cf. [6, $(9.3 .7)]$.

4.7. Theorem. If $R$ is a noetherian ring, and $M$ is a finite module of finite CIdimension, then for each $R$-module $N$ the following conditions are equivalent.

(i) $\operatorname{Ext}_{R}^{n}(M, N)=0$ for $\mathrm{cx}_{R} M+1$ consecutive values of $n>\mathrm{CI}-\operatorname{dim}_{R} M$.

(ii) $\operatorname{Ext}_{R}^{n}(M, N)=0$ for $n \gg 0$.

(iii) $\operatorname{Ext}_{R}^{n}(M, N)=0$ for all $n>$ CI-dim $R$ M.

(iv) $\widehat{\operatorname{Ext}}_{R}^{n}(M, N)=0$ for $\mathrm{cx}_{R} M+1$ consecutive values of $n$.

(v) $\widehat{\operatorname{Ext}}_{R}^{n}(M, N)=0$ for $n \ll 0$.

(vi) $\widehat{\operatorname{Ext}}_{R}^{n}(M, N)=0$ for all $n \in \mathbb{Z}$. 
Proof. To simplify notation, we set $r=$ CI-dim $R$ and $c=\operatorname{cx}_{R} M$.

If $\mathfrak{m}$ is a maximal ideal of $R$, then CI- $\operatorname{dim}_{R_{\mathfrak{m}}} M_{\mathfrak{m}} \leq r$ and $\operatorname{cx}_{R_{\mathfrak{m}}} M_{\mathfrak{m}} \leq c$, while $\operatorname{Ext}_{R_{\mathfrak{m}}}^{n}\left(M_{\mathfrak{m}}, N_{\mathfrak{m}}\right) \cong \operatorname{Ext}_{R}^{n}(M, N)_{\mathfrak{m}}$ and $\widehat{\operatorname{Ext}}_{R_{\mathfrak{m}}}^{n}\left(M_{\mathfrak{m}}, N_{\mathfrak{m}}\right) \cong \widehat{\operatorname{Ext}}_{R}^{n}(M, N)_{\mathfrak{m}}$ hold for each $n \in \mathbb{Z}$. Thus, to prove the theorem we may assume that $R$ is local. In that case, by 4.1.2 and 4.1.3 we may further assume that $R=Q /(\boldsymbol{f})$ where $Q$ is local, $f$ is a $Q$-regular sequence of length $c=\operatorname{cx}_{R} M$, and proj $\operatorname{dim}_{Q} M$ is finite.
Clearly (iii) $\Longrightarrow$
(ii) $\Longrightarrow$
(i). Next we establish
(i) $\Longrightarrow$
(iii):

Let $s$ be the smallest index $n>r$ that appears in a block of at least $(c+1)$ consecutive vanishing $\operatorname{Ext}_{R}^{n}(M, N)$, and let $t \leq \infty$ be the largest index in that block. The Cartan-Eilenberg change of rings spectral sequence [14, XVI §5] has

$$
{ }^{2} \mathrm{E}^{p, q}=\operatorname{Ext}_{R}^{p}\left(M, \operatorname{Ext}_{Q}^{q}(R, N)\right) \Longrightarrow \operatorname{Ext}_{Q}^{p+q}(M, N)
$$

Computing with the Koszul complex $\boldsymbol{K}$ that resolves $R$ over $Q$ we get

$$
\operatorname{Ext}_{Q}^{q}(R, N)=\mathrm{H}^{q} \operatorname{Hom}(\boldsymbol{K}, N)=\operatorname{Hom}\left(K_{q}, N\right)=N^{\left(\begin{array}{c}
c \\
q
\end{array}\right)},
$$

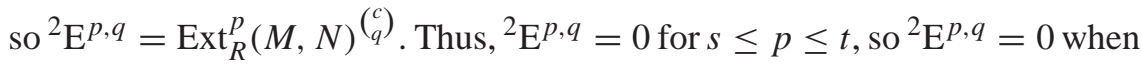
$s+c \leq p+q \leq t$, and the only possibly non-zero module with $p+q=s+c-1$ (respectively, $p+q=t+1$ ) is ${ }^{2} \mathrm{E}^{s-1, c}$ (respectively, ${ }^{2} \mathrm{E}^{t+1,0}$ ). For degree reasons, no non-trivial differential can enter or exit these modules, hence

$$
\begin{gathered}
\operatorname{Ext}_{Q}^{s+c-1}(M, N) \cong{ }^{2} \mathrm{E}^{s-1, c}=\operatorname{Ext}_{R}^{s-1}(M, N) ; \\
\operatorname{Ext}_{Q}^{t+1}(M, N) \cong{ }^{2} \mathrm{E}^{t+1,0}=\operatorname{Ext}_{R}^{t+1}(M, N) .
\end{gathered}
$$

Using, in turn, the Auslander-Buchsbaum Equality, the invariance of depth under finite maps, and 4.1.5 we obtain the following equalities

$$
\operatorname{proj}_{\operatorname{dim}_{Q}} M=\operatorname{depth} Q-\operatorname{depth}_{Q} M=\operatorname{depth} R+c-\operatorname{depth}_{R} M=r+c .
$$

If $t<\infty$, then $t+1 \geq s+c+1 \geq r+c+2>\operatorname{proj}_{\operatorname{dim}_{Q}} M$, $\operatorname{soExt}_{R}^{t+1}(M, N) \cong$ $\operatorname{Ext}_{Q}^{t+1}(M, N)=0$, contrary to the maximality of $t$; thus, $t=\infty$. If $s>r+1$, then $s+c-1>r+c=\operatorname{proj}_{\operatorname{dim}_{Q}} M$, so $\operatorname{Ext}_{Q}^{s+c-1}(M, N)=0$, hence $\operatorname{Ext}_{R}^{s-1}(M, N)=0$; this contradicts the minimality of $s$, so $s=r+1$.

The implications (vi) $\Longrightarrow(\mathrm{v}) \Longrightarrow$ (iv) are clear. The isomorphisms in 4.4 .6 yield (i) $\Longrightarrow$ (iv) and (vi) $\Longrightarrow$ (ii). To finish the proof, we show (iv) $\Longrightarrow$ (vi):

Fix an integer $n$. By hypothesis, there is an integer $s$ such that $\widehat{\operatorname{Ext}}_{R}^{i}(M, N)$ $=0$ for $s \leq i \leq s+c$. Since $\mathrm{G}-\operatorname{dim}_{R} M=r$ by 4.4.3, the module $M$ has a complete resolution $\boldsymbol{T}$ by 4.4.4. Choosing an integer $u>-\min \{n, s\}$, set $L=$ $\Omega^{u} \boldsymbol{T}$ and note that CI- $\operatorname{dim}_{R} L=0$ by Proposition 4.5.2. The isomorphisms 
$\widehat{\operatorname{Ext}}_{R}^{j}(L, N) \cong \widehat{\operatorname{Ext}}_{R}^{j-u}(M, N)$ of 4.4.2 show that $\widehat{\operatorname{Ext}}_{R}^{j}(L, N)$ vanishes for $c+1$ consecutive values of $j>0$. As $\widehat{E x t}_{R}^{j}(L, N) \cong \operatorname{Ext}_{R}^{j}(L, N)$ for $j>0$ by 4.4.6, the already proved implication (i) $\Longrightarrow$ (iii) shows that $\operatorname{Ext}_{R}^{j}(L, N)=0$ for $j>0$, in particular $\widehat{\operatorname{Ext}}_{R}^{n}(M, N) \cong \operatorname{Ext}_{R}^{n+u}(L, N)=0$. As $n$ was arbitrary, we are done.

The preceding theorem is complemented by the following non-vanishing result, due to Araya and Yoshino [1, (4.2), (4,4)].

4.8. Proposition. Let $M, N$ be finite non-zero modules over a local ring $R$ with $\operatorname{Ext}_{R}^{n}(M, N)=0$ for $n \gg 0$. If CI- $\operatorname{dim}_{R} M=r<\infty$, then $\operatorname{Ext}_{R}^{r}(M, N) \neq 0$, and

$$
\operatorname{proj} \operatorname{dim}_{R} M=\sup \left\{n \in \mathbb{N} \mid \operatorname{Ext}_{R}^{n}(M, M) \neq 0\right\} .
$$

Proof. As in the preceding proof, we may assume that $R=Q /(\boldsymbol{f})$ where $Q$ is a local ring with maximal ideal $\mathfrak{n}, \boldsymbol{f}$ is a $Q$-regular sequence of length $c=\operatorname{cx}_{R} M$, and proj $\operatorname{dim}_{Q} M$ finite. In that proof, the argument for (i) $\Longrightarrow$ (iii) showed that $\operatorname{proj}_{\operatorname{dim}_{Q}} M=r+c$ and $\operatorname{Ext}_{R}^{r}(M, N) \cong C$ with $C=\operatorname{Ext}_{Q}^{r+c}(M, N)$. If $\boldsymbol{E}$ is a minimal free resolution of $M$ over $Q$, then $C$ is the cokernel of the homomorphism

$$
\operatorname{Hom}\left(\partial_{c+r}^{E}, N\right): \operatorname{Hom}_{Q}\left(E_{c+r-1}, N\right) \longrightarrow \operatorname{Hom}_{Q}\left(E_{c+r}, N\right)
$$

whose image is in $\mathfrak{n} \operatorname{Hom}_{Q}\left(E_{c+r}, N\right)$; when $N$ is finite $C \neq 0$ by Nakayama.

Set $p=\operatorname{proj}_{\operatorname{dim}_{R}} M$ and $q=\sup \left\{n \in \mathbb{N} \mid \operatorname{Ext}_{R}^{n}(M, M) \neq 0\right\}$. If $p$ is infinite, then so is $q$ by Theorem 4.2. If $p$ is finite, then $p=r$ by 4.5.1, and $q=r$ by the already established part of the proposition.

The equivalence of the first three conditions below slightly extends a result of Jorgensen [24, (2.1)], cf. [6, (9.3.6)] for a concise proof. Again, the number of consecutive vanishing Tor's in (i) or (iv) may not be decreased, cf. $[24,(4.1)]$.

4.9. Theorem. If $R$ is a noetherian ring, and $M$ is a finite module of finite $C I$ dimension, then for each $R$-module $N$ the following conditions are equivalent.

(i) $\operatorname{Tor}_{n}^{R}(M, N)=0$ for $\mathrm{cx}_{R} M+1$ consecutive values of $n>\mathrm{CI}-\operatorname{dim}_{R} M$.

(ii) $\operatorname{Tor}_{n}^{R}(M, N)=0$ for $n \gg 0$.

(iii) $\operatorname{Tor}_{n}^{R}(M, N)=0$ for all $n>$ CI-dim ${ }_{R} M$.

(iv) $\widehat{\operatorname{Tor}}_{n}^{R}(M, N)=0$ for $\mathrm{cx}_{R} M+1$ consecutive values of $n$.

(v) $\widehat{\operatorname{Tor}}_{n}^{R}(M, N)=0$ for $n \ll 0$.

(vi) $\widehat{\operatorname{Tor}}_{n}^{R}(M, N)=0$ for all $n \in \mathbb{Z}$.

Proof. A transposition of the proof of Theorem 4.7 yields the desired result. 


\section{Complete intersections}

By definition, a local ring $R$ with maximal ideal $\mathfrak{m}$ is a complete intersection if in some Cohen presentation of its $\mathfrak{m}$-adic completion $\widehat{R}$ as a residue ring of a complete regular local ring $Q$, the defining ideal $\mathfrak{a}$ is generated by a regular sequence. It is well known that the defining ideal of any Cohen presentation of $\widehat{R}$ then has this property, cf. 5.10.2, and the least length of such a sequence is equal to $\operatorname{codim} R$.

The results of the preceding section fully apply to complete intersections, due to

5.1. Let $M$ be a finite $R$-module. If $R$ is a local complete intersection, then proj $\operatorname{dim}_{Q}\left(M \otimes_{R} \widehat{R}\right)<\infty$ for any quasi-deformation $R \rightarrow \widehat{R} \leftarrow Q$ with regular ring $Q$, so CI-dim $R=\operatorname{depth} R-\operatorname{depth}_{R} M \leq \operatorname{depth} R \leq \operatorname{dim} R$ by 4.1.5.

In this section we attach to any pair of finite modules $M, N$ over a local complete intersection $R$ a variety $\mathrm{V}_{R}^{*}(M, N)$, derive some of its fundamental properties, and apply them to prove the results described in the introduction.

5.2. Let $R$ be a local complete intersection with residue field $k$.

We fix an embedding of $k$ into an algebraic closure, $\widetilde{k}$.

For any pair of finite $R$-modules $M, N$ we define its support variety by

$$
\mathrm{V}_{R}^{*}(M, N)=\mathrm{V}(Q, \widehat{R} ; \widehat{M}, \widehat{N}) \subseteq(\mathfrak{a} / \mathfrak{n} \mathfrak{a}) \otimes_{k} \widetilde{k} .
$$

where $\widehat{R} \cong Q / \mathfrak{a}$ is a Cohen presentation with a complete regular local ring $Q$, and $\mathrm{V}(Q, \widehat{R} ; \widehat{M}, \widehat{N})$ is the variety defined in Remark 2.3 .

Varieties of pairs do not depend on the choice of the Cohen presentation, due to

5.3. Theorem. The variety $\mathrm{V}(Q, \widehat{R} ; \widehat{M}, \widehat{N})$ is contained in $\left(\left(\mathfrak{a} \cap \mathfrak{n}^{2}\right) / \mathfrak{n a}\right) \otimes_{k} \widetilde{k}$.

Any presentation $\widehat{R} \cong Q^{\prime} / \mathfrak{a}^{\prime}$ with a complete regular local ring $\left(Q^{\prime}, \mathfrak{n}^{\prime}, k\right)$ defines a k-linear isomorphism $\varkappa:\left(\mathfrak{a}^{\prime} \cap \mathfrak{n}^{\prime 2}\right) / \mathfrak{n}^{\prime} \mathfrak{a}^{\prime} \cong\left(\mathfrak{a} \cap \mathfrak{n}^{2}\right) / \mathfrak{n} \mathfrak{a}$, and $\varkappa \otimes_{k} \widetilde{k}$ maps $\mathrm{V}\left(Q^{\prime}, \widehat{R} ; \widehat{M}, \widehat{N}\right)$ bijectively onto $\mathrm{V}(Q, \widehat{R} ; \widehat{M}, \widehat{N})$ for all $R$-modules $M, N$.

We recall the construction of a variety $\mathrm{V}_{R}^{*}(M)$, given in [5, §6].

5.4. For any local ring $R$ with residue field $k$ the algebra $\operatorname{Ext}_{R}^{*}(k, k)$ (under composition products) is the universal enveloping algebra of a graded Lie algebra $\pi^{*}(R)$, cf. [6, §10] for details of the construction.

If $R$ is a complete intersection, then $\pi^{n}(R)=0$ for $n \geq 3$, so $\pi^{2}(R)$ is a central Lie subalgebra and $\operatorname{rank}_{k} \pi^{2}(R)=\operatorname{codim} R$. By the PoincaréBirkhoff-Witt Theorem for graded Lie algebras, the associative subalgebra $\mathcal{P}=k\left[\pi^{2}(R)\right] \subseteq \operatorname{Ext}_{R}^{*}(k, k)$ is a polynomial ring on variables of degree 2 . When $M$ is a finite $R$-module, $\operatorname{Ext}_{R}^{*}(M, k)$ is a finite module over $\mathcal{P}$. In [5, (6.2)] the cohomological variety $\mathrm{V}_{R}^{*}(M)$ is defined to be the zero-set over $\widetilde{k}$ of the annihilator of $\operatorname{Ext}_{R}^{*}(M, k)$ in $\mathcal{P}$. 
5.5. Theorem. The varieties $\mathrm{V}(Q, \widehat{R} ; \widehat{M}, k)$ and $\mathrm{V}_{R}^{*}(M)$ are isomorphic.

In our main result we establish basic properties of support varieties of pairs, and some new properties of varieties of modules. Theorems I and II in the introduction are abstracted from the next couple of results.

5.6. Theorem. If $(R, \mathfrak{m}, k)$ is a local complete intersection of codimension $c$, and $M, N$ are finite $R$-modules, then the following hold.

(1) $\mathrm{V}_{R}^{*}(M, N)$ is a cone in $\tilde{k}^{c}$.

(2) $\operatorname{dim} \mathrm{V}_{R}^{*}(M, N)=\operatorname{cx}_{R}(M, N)$.

(3) $\mathrm{V}_{R}^{*}(M, N)=\{0\}$ if and only if $\operatorname{Ext}_{R}^{n}(M, N)=0$ for $n \gg 0$, if and only if $\widehat{\operatorname{Ext}}_{R}^{n}(M, N)=0$ for all $n \in \mathbb{Z}$.

(4) $\operatorname{dim} \mathrm{V}_{R}^{*}(M, N) \leq 1$ if and only if $\operatorname{Ext}_{R}^{n}(M, N) \cong \operatorname{Ext}_{R}^{n+2}(M, N)$ for all $n \gg 0$.

(5) $\mathrm{V}_{R}^{*}(M, N)=\mathrm{V}_{R}^{*}\left(M^{\prime}, N^{\prime}\right)$ if $M^{\prime}$ is a syzygy of $M$ and $N^{\prime}$ is a syzygy of $N$.

(6) If $0 \rightarrow M_{1} \rightarrow M_{2} \rightarrow M_{3} \rightarrow 0$ and $0 \rightarrow N_{1} \rightarrow N_{2} \rightarrow N_{3} \rightarrow 0$ are exact sequences of finite $R$-modules, then for $\{h, i, j\}=\{1,2,3\}$ there are inclusions

$$
\begin{aligned}
& \mathrm{V}_{R}^{*}\left(M_{h}, N\right) \subseteq \mathrm{V}_{R}^{*}\left(M_{i}, N\right) \cup \mathrm{V}_{R}^{*}\left(M_{j}, N\right) ; \\
& \mathrm{V}_{R}^{*}\left(M, N_{h}\right) \subseteq \mathrm{V}_{R}^{*}\left(M, N_{i}\right) \cup \mathrm{V}_{R}^{*}\left(M, N_{j}\right) .
\end{aligned}
$$

(7) $\mathrm{V}_{R}^{*}(M, N)=\bigcup_{i, j} \mathrm{~V}_{R}^{*}\left(M_{i}, N_{j}\right)$ if $M=\bigoplus_{i=1}^{r} M_{i}$ and $N=\bigoplus_{j=1}^{s} N_{j}$.

(8) $\mathrm{V}_{R}^{*}(M, N)=\mathrm{V}_{R}^{*}(M) \cap \mathrm{V}_{R}^{*}(N)=\mathrm{V}_{R}^{*}(N, M)$.

(9) $\mathrm{V}_{R}^{*}(M, M)=\mathrm{V}_{R}^{*}(M, k)=\mathrm{V}_{R}^{*}(k, M)=\mathrm{V}_{R}^{*}(M)$.

(10) $\mathrm{V}_{R}^{*}(M)=\mathrm{V}_{R}^{*}\left(\operatorname{Ext}_{R}^{m}(M, R)\right)$ if $M$ is Cohen-Macaulay of codimension $\mathrm{m}$.

By Part (2) of the theorem, the following relations come from equalities of varieties and estimates for the dimension of intersection of varieties in (9) and (8).

5.7. Corollary. If $M$ is a finite modules over a local complete intersection $R$, then

$$
\mathrm{cx}_{R}(M, M)=\mathrm{cx}_{R} M
$$

and for each finite $R$-module $N$ there are (in)equalities

$$
\operatorname{cx}_{R} M+\operatorname{cx}_{R} N-c \leq \operatorname{cx}_{R}(M, N)=\operatorname{cx}_{R}(N, M) \leq \min \left\{\operatorname{cx}_{R} M, \operatorname{cx}_{R} N\right\} .
$$


As another consequence, we get equations for the support variety of a pair. They encode the asymptotic behavior of $\operatorname{Ext}_{R}^{*}(M, N)$ into finitely many matrices of systems of higher homotopies $\sigma$ and $\tau$, defined over the regular ring $Q$. These homotopies can be computed using Grayson and Stillman's software system Macaulay 2 [18].

5.8. Corollary. Assume that $R=Q /(\boldsymbol{f})$ for a regular local ring $Q$ and $a$ $Q$-regular sequence $\boldsymbol{f}=f_{1}, \ldots, f_{c}$. Let $\boldsymbol{E}$ be a free resolution of $M$ over $Q$ with $E_{n}=0$ for $n>2 u+1$, let $\boldsymbol{F}$ be a free resolution of $N$ over $Q$ with $F_{n}=0$ for $n>2 v+1$, set $r=\sum_{i=0}^{u} \operatorname{rank}_{Q} E_{2 i}$ and $s=\sum_{i=0}^{v} \operatorname{rank}_{Q} F_{2 i}$, and fix systems of higher homotopies for $\boldsymbol{f}$, say $\boldsymbol{\sigma}$ on $\boldsymbol{E}$ and $\boldsymbol{\tau}$ on $\boldsymbol{F}$, respectively.

If matrices $\bar{\sigma}(x)$ and $\bar{\tau}(x)$ over $k\left[x_{1}, \ldots, x_{c}\right]$ are formed from $\boldsymbol{\sigma}$ and $\boldsymbol{\tau}$ as in Theorem 3.2, then $\mathrm{V}_{R}^{*}(M, N)$ is the zero-set of the ideal $I_{r}(\bar{\sigma}(x))+I_{S}(\bar{\tau}(x))$, where $I_{t}(\gamma)$ denotes the ideal generated by the $t \times t$ minors of a matrix $\gamma$.

Proof. By Theorem 3.2 the zero set of $I_{r}(\bar{\sigma}(x))$ is $\mathrm{V}_{R}^{*}(M)$, and that of $I_{S}(\bar{\tau}(x))$ is $\mathrm{V}_{R}^{*}(N)$. Thus, the zero set of $I_{r}(\bar{\sigma}(x))+I_{S}(\bar{\tau}(x))$ is equal to $\mathrm{V}_{R}^{*}(M) \cap \mathrm{V}_{R}^{*}(N)$. By part (8) of Theorem 5.6 the intersection coincides with $\mathrm{V}_{R}^{*}(M, N)$.

We start working on the proof of the theorems with

5.9. Lemma. Let $(Q, \mathfrak{n}, k)$ and $\left(Q^{\prime}, \mathfrak{n}^{\prime}, k\right)$ be complete regular local rings, and let $Q^{\prime} \stackrel{\kappa}{\longrightarrow} Q \stackrel{\rho}{\longrightarrow} \widehat{R}$ be surjective homomorphisms with $\mathfrak{a}=\operatorname{Ker} \rho$ and $\mathfrak{a}^{\prime}=\operatorname{Ker}(\rho \kappa)$.

(1) The induced k-linear map $\bar{\kappa}:\left(\mathfrak{a}^{\prime} \cap \mathfrak{n}^{\prime 2}\right) / \mathfrak{n}^{\prime} \mathfrak{a}^{\prime} \rightarrow\left(\mathfrak{a} \cap \mathfrak{n}^{2}\right) / \mathfrak{n a}$ is bijective.

(2) $\operatorname{rank}_{k}\left(\mathfrak{a} \cap \mathfrak{n}^{2}\right) / \mathfrak{n} \mathfrak{a} \geq \operatorname{codim} R$.

(3) equality holds in (2) if and only if $\mathfrak{a}$ is generated by a regular sequence.

Proof. (1) The homomorphism $\kappa$ defines a commutative diagram of $Q^{\prime}$-modules

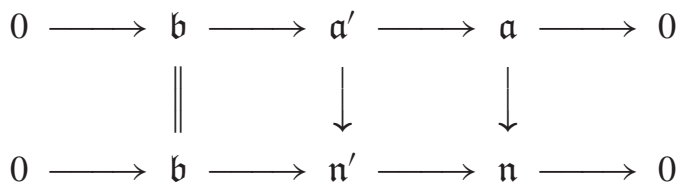

where the rows are exact and $\mathfrak{b}=\operatorname{Ker} \kappa$. Tensoring it over $Q^{\prime}$ with $k$ we get a commutative diagram of $k$-vector spaces with exact rows and columns: 


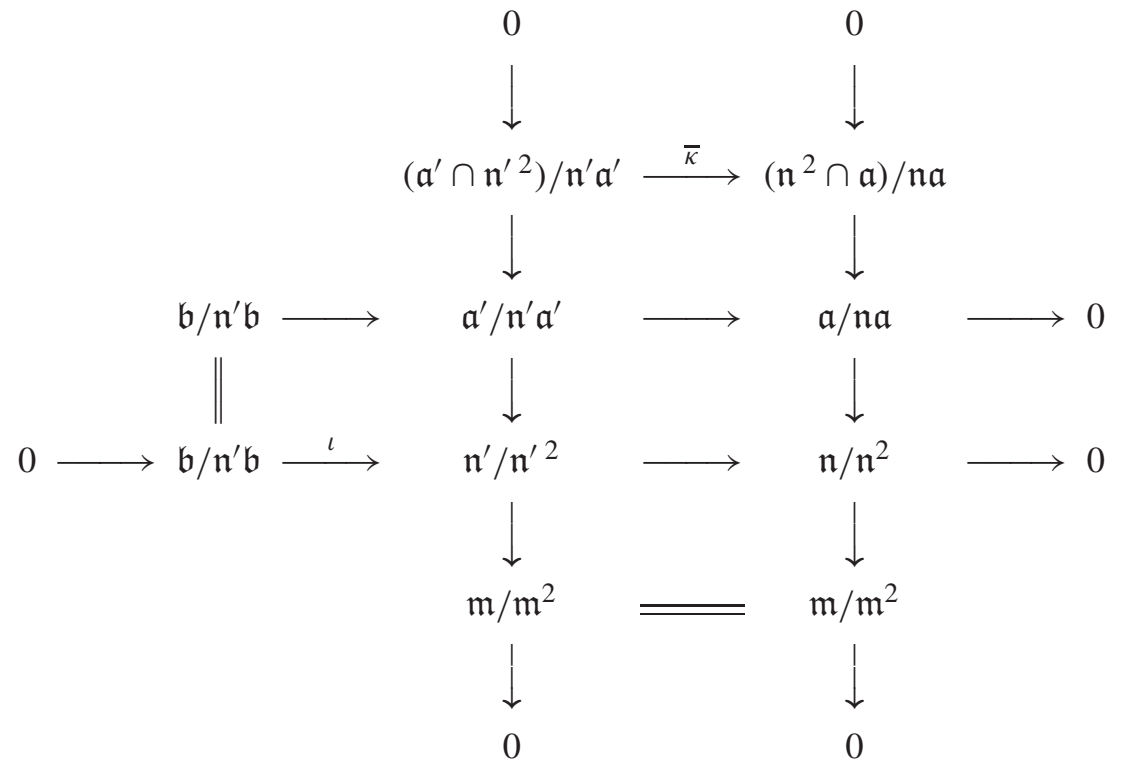

Indeed, the only point at stake is the injectivity of $\iota$ it is due to the fact that $\mathfrak{b}$, being the kernel of a surjection of regular local rings, is generated by part of a regular system of parameters for $Q$. The Snake Lemma shows that $\bar{\kappa}$ is bijective.

(2) Let $\mathfrak{m}$ be the maximal ideal of $R$. The inequality comes from the relations

$$
\begin{aligned}
v_{R}(\mathfrak{m})-\operatorname{rank}_{k}\left(\mathfrak{a} \cap \mathfrak{n}^{2}\right) / \mathfrak{n} \mathfrak{a} & =v_{Q}(\mathfrak{n})-v_{Q}(\mathfrak{a})=\operatorname{dim} Q-v_{Q}(\mathfrak{a}) \\
& \geq \operatorname{dim} Q-\operatorname{height}(\mathfrak{a})=\operatorname{dim} \widehat{R}=\operatorname{dim} R
\end{aligned}
$$

due, in turn, to the right hand column, the regularity of $Q$, the Krull Principal Ideal Theorem, the catenarity of $Q$, and the invariance of dimension under completion.

(3) The same chain shows that $\operatorname{rank}_{k}\left(\mathfrak{a} \cap \mathfrak{n}^{2}\right) / \mathfrak{n} \mathfrak{a}=\operatorname{codim} R$ if and only if height $(\mathfrak{a})=v_{Q}(\mathfrak{a})$. By the Cohen-Macaulay Theorem, the last equality holds if and only if $\mathfrak{a}$ can be generated by a $Q$-regular sequence.

The preceding lemma is best used in conjunction with a construction of Grothendieck [20] that compares different Cohen presentations.

5.10. Let $(Q, \mathfrak{n}, k)$ and $\left(Q^{\prime}, \mathfrak{n}^{\prime}, k\right)$ be complete regular local rings, $\rho: Q \rightarrow \widehat{R}$ and $\rho^{\prime}: Q^{\prime} \rightarrow \widehat{R}$ be surjective homomorphisms of rings.

5.10.1. There exists a complete regular local ring $P$ with surjective homomorphisms $Q^{\prime} \stackrel{\kappa^{\prime}}{\longleftarrow} P \stackrel{\kappa}{\longrightarrow} Q$ such that $\rho^{\prime} \kappa^{\prime}=\rho \kappa$.

Indeed, the fiber product $Q^{\prime} \times{ }_{\widehat{R}} Q$ is a complete local ring, cf. [20, (19.3.2.1)], so take a Cohen presentation of $Q^{\prime} \times \widehat{R} Q$ as homomorphic image of a regular local ring $P$ and get the desired homomorphisms as compositions of canonical projections. 
As $\kappa$ is a surjection of regular local rings, any minimal system of generators of $\operatorname{Ker} \kappa$ can be extended to a minimal system of generators of the maximal ideal of $P$, and similarly for $\operatorname{Ker} \kappa^{\prime}$. In view of Lemma 5.9.3, we get

5.10.2. The ideal $\mathfrak{a}=\operatorname{Ker} \rho$ is generated by a regular sequence if and only if the ideal $\mathfrak{a}^{\prime}=\operatorname{Ker} \rho^{\prime}$ is generated by a regular sequence.

Proof of Theorem 5.5. Let $M$ be a finite module over a local complete intersection $R$, and let $\widehat{R} \cong Q / \mathfrak{a}$ be a Cohen presentation. The $R$-flatness of $\widehat{R}$ yields a canonical isomorphisms $\operatorname{Ext}_{\widehat{R}}^{*}(\widehat{M}, k) \cong \operatorname{Ext}_{R}^{*}(M, k)$ compatible with an isomorphism of graded algebras $\operatorname{Ext}_{\widehat{R}}^{*}(k, k) \cong \operatorname{Ext}_{R}^{*}(k, k)$ that maps $\pi^{*}(\widehat{R})$ to $\pi^{*}(R)$. It follows that $\mathrm{V}_{\widehat{R}}^{*}(\widehat{M}) \cong \mathrm{V}_{R}^{*}(M)$, cf. 5.4 , so we may assume that $R=\widehat{R} \cong Q / \mathfrak{a}$ and $M=\widehat{M}$.

Choose a regular sequence $\boldsymbol{f}$ generating $\mathfrak{a}$ by first picking $\left\{f_{1}, \ldots, f_{b}\right\}$ to map onto a basis of $\mathfrak{a} /\left(\mathfrak{a} \cap \mathfrak{n}^{2}\right) \cong\left(\mathfrak{a}+\mathfrak{n}^{2}\right) / \mathfrak{n}^{2}$, then extending it by elements $f_{b+1}, \ldots, f_{c} \in \mathfrak{a} \cap \mathfrak{n}^{2}$ to a minimal set of generators of $\mathfrak{a}$. Also, choose a minimal set of generators $\boldsymbol{f}^{\prime}=\left\{f_{1}^{\prime}, \ldots, f_{c^{\prime}}^{\prime}\right\}$ of $\mathfrak{n}^{\prime}$ with $f_{j}^{\prime}=f_{j}$ for $1 \leq$ $j \leq b$. Since $Q$ is regular, $\boldsymbol{f}^{\prime}$ is a $Q$-regular sequence, so Proposition 1.2 yields an isomorphism $\operatorname{Ext}_{R}^{*}(k, k) \cong \overline{\mathcal{R}} \otimes_{k} \bigwedge_{k}\left(k^{c^{\prime}-b}\right)$ of graded modules over $\mathcal{R}=s \otimes_{R} k=k\left[\chi_{1}, \ldots, \chi_{c}\right]$, where $s$ is the ring of cohomology operators defined by $\boldsymbol{f}$ and $\overline{\mathcal{R}}=\mathcal{R} /\left(\chi_{1}, \ldots, \chi_{b}\right)$.

Let $\xi_{k}: \mathcal{R} \rightarrow \operatorname{Ext}_{R}^{*}(k, k)$ be the homomorphism of graded $k$-algebras from 1.1.2. The preceding computation yields $\operatorname{Ker} \xi_{k}=\left(\chi_{1}, \ldots, \chi_{b}\right)$. By [9, (5.2)] each $\xi_{k}\left(\chi_{j}\right) \in \operatorname{Ext}_{R}^{2}(k, k)$ annihilates the subspace of $\operatorname{Tor}_{2}^{R}(k, k)$ spanned by products of elements in $\operatorname{Tor}_{1}^{R}(k, k)$; this property characterizes the elements of $\pi^{2}(R)$, cf. [6, $\left.\S 10.2\right]$, so $\xi_{k}$ induces an injective homomorphism of graded $k$-algebras $\bar{\xi}: \overline{\mathcal{R}} \rightarrow \mathcal{P}$. Both are generated in degree two. Also, $\operatorname{rank}_{k} \overline{\mathcal{R}}^{2}=$ $c-b=\operatorname{codim} R$ by Lemma 5.9.2 and $\operatorname{rank}_{k} \mathcal{P}^{2}=\operatorname{rank}_{k} \pi^{2}(R)=\operatorname{codim} R$ by $[6,(10.2 .1)$ and (7.5.1)], so $\bar{\xi}$ is bijective. By 1.1.2 the action of $\mathcal{R}$ on $\mathscr{E}=$ $\operatorname{Ext}_{R}^{*}(M, k)$ factors through $\xi_{k}$, hence it induces an isomorphism $\mathcal{R} / \operatorname{ann}_{\mathcal{R}} \mathscr{E} \cong$ $\mathcal{P} / \operatorname{ann}_{\mathcal{P}} \mathcal{E}$, so $\mathrm{V}_{R}^{*}(M) \cong \mathrm{V}(Q, \boldsymbol{f} ; M)$.

We recall a change of rings theorem of Nagata [29, (27.3)], cf. also [6, (3.3.5.1)].

5.11. If $\left(Q^{\prime}, \mathfrak{n}^{\prime}\right)$ is a local ring, $g^{\prime}$ a is non-zero-divisor in $\mathfrak{n}^{\prime} \backslash \mathfrak{n}^{\prime 2}$, and $M \neq 0$ is a finite $Q^{\prime}$-module with $g^{\prime} M=0$, then $\operatorname{proj}_{\operatorname{dim}_{Q^{\prime}}}(M)=1+$ proj $\operatorname{dim}_{Q^{\prime} /\left(g^{\prime}\right)}(M)$.

The next result is a counterpart in cohomology of the theorem of Huneke and Wiegand [22, (1.9)] that the vanishing of two consecutive Tor's over a local hypersurface implies that one of the modules involved has finite projective dimension. 
5.12. Proposition. If $M, N$ are finite modules over a local hypersurface $R$, and

$$
\operatorname{Ext}_{R}^{n}(M, N)=\operatorname{Ext}_{R}^{n+1}(M, N)=0
$$

for some $n>\operatorname{dim} R$, then $\operatorname{proj} \operatorname{dim}_{R} M<\infty$ or $\operatorname{proj}_{\operatorname{dim}} N<\infty$.

Proof. By 4.7 the hypothesis implies that $\operatorname{Ext}_{R}^{n}(M, N)=0$ for all $n>\operatorname{dim} R$. Replacing $M$ by a high enough syzygy, we may assume that $\operatorname{Ext}_{R}^{n}(M, N)=0$ for $n>0$. Completing if necessary, we may further assume that $R \cong Q /(f)$ for a regular local ring $Q$ and a non-zero-divisor $f$. If both $\operatorname{proj}^{\operatorname{dim}_{R}} M$ and inj $\operatorname{dim}_{R} N$ were infinite, then by Corollary 1.4 and Proposition 1.5.3 we would have

$$
1 \geq \mathrm{px}_{R} \operatorname{Hom}_{R}(M, N)=\mathrm{cx}_{R} M+\mathrm{px}_{R} N \geq 1+1 .
$$

This is absurd, so (at least) one homological dimension is finite. Hypersurfaces being Gorenstein rings, inj $\operatorname{dim}_{R} N$ is finite if and only if $\operatorname{proj}^{\operatorname{dim}_{R} N} N$ is.

Proof of Theorem 5.3. Clearly, we may replace $R$ by its completion, and so write $M$ and $N$ instead of $\widehat{M}$ and $\widehat{N}$. By 5.10 .1 we may assume that there is a surjective homomorphism $\kappa: Q^{\prime} \rightarrow Q$ of regular local rings. Any minimal system of generators $\boldsymbol{g}$ of $\operatorname{Ker} \kappa$ can be extended to a regular system of parameters for $Q$, so the ring $Q^{\prime} /\left(\boldsymbol{g}^{\prime}\right)$ is regular for each subset $\boldsymbol{g}^{\prime}$ of $\boldsymbol{g}$. Inducing on the cardinality of $\boldsymbol{g}$, we may assume that $Q=Q^{\prime} /\left(g^{\prime}\right)$ for some $g^{\prime} \in \mathfrak{n}^{\prime} \backslash \mathfrak{n}^{\prime 2}$. Applying 2.2 to $Q^{\prime}$ we may further assume that the common residue field $k$ of $Q^{\prime}, Q$, and $R$ is algebraically closed.

If $\bar{f} \in \mathfrak{a} / \mathfrak{n a}$ is not contained in the subspace $\left(\mathfrak{a} \cap \mathfrak{n}^{2}\right) / \mathfrak{n a}$, and $f \in \mathfrak{n}$ is a lifting of $\bar{f}$ to $\mathfrak{n}$, then $f \notin \mathfrak{n}^{2}$. It follows that $Q /(f)$ is regular, hence $\operatorname{Ext}_{Q /(f)}^{n}(M, N)=0$ for $n \gg 0$, so $\bar{f} \notin \mathrm{V}(Q, f ; M, N)$ by Theorem 2.5 . Thus, $\mathrm{V}(Q, \boldsymbol{f} ; M, N) \subseteq\left(\mathfrak{a} \cap \mathfrak{n}^{2}\right) / \mathfrak{n} \mathfrak{a}$.

Lemma 5.9.1 yields a $k$-linear isomorphism $\bar{\kappa}:\left(\mathfrak{a}^{\prime} \cap \mathfrak{n}^{\prime 2}\right) / \mathfrak{n}^{\prime} \mathfrak{a}^{\prime} \cong$ $\left(\mathfrak{a} \cap \mathfrak{n}^{2}\right) / \mathfrak{n} \mathfrak{a}$. We want to prove that $\overline{f^{\prime}} \in\left(\mathfrak{a}^{\prime} \cap \mathfrak{n}^{\prime 2}\right) / \mathfrak{n}^{\prime} \mathfrak{a}^{\prime}$ is in $\mathrm{V}\left(Q^{\prime}, \boldsymbol{f}^{\prime} ; M, N\right)$ if and only if $\bar{f}=\bar{\kappa}\left(\overline{f^{\prime}}\right) \in\left(\mathfrak{a} \cap \mathfrak{n}^{2}\right) / \mathfrak{n} \mathfrak{a}$ is in $\mathrm{V}(Q, \boldsymbol{f} ; M, N)$. If $f^{\prime} \in \mathfrak{a}^{\prime} \cap \mathfrak{n}^{\prime 2}$ is a lifting of $\bar{f}^{\prime}$, then $f=\kappa\left(f^{\prime}\right) \in \mathfrak{a} \cap \mathfrak{n}^{2}$ is a lifting of $\bar{f}$, so by Theorem 2.5 it suffices to prove that $\operatorname{Ext}_{Q^{\prime} /\left(f^{\prime}\right)}^{n}(M, N)=0$ for $n \gg 0$ if and only if $\operatorname{Ext}_{Q /(f)}^{n}(M, N)=0$ for $n \gg 0$.

The sequence $f^{\prime}, g^{\prime}$ is $Q^{\prime}$-regular, so the image $g^{\prime \prime}$ of $g^{\prime}$ in $Q^{\prime \prime}=Q^{\prime} /\left(f^{\prime}\right)$ is a non-zero-divisor. The change of rings spectral sequence with second page

$$
{ }^{2} \mathrm{E}^{p, q}=\operatorname{Ext}_{Q^{\prime \prime} /\left(g^{\prime \prime}\right)}^{p}\left(M, \operatorname{Ext}_{Q^{\prime \prime}}^{q}\left(Q^{\prime \prime} /\left(g^{\prime \prime}\right), N\right)\right) \Longrightarrow \operatorname{Ext}_{Q^{\prime \prime}}^{p+q}(M, N)
$$

has ${ }^{2} \mathrm{E}^{p, q}=0$ unless $q \neq 0,1$, so it degenerates into a long exact sequence. Due to the isomorphisms $Q^{\prime \prime} /\left(g^{\prime \prime}\right) \cong Q^{\prime} /\left(f^{\prime}, g^{\prime}\right) \cong Q /(f)$ it can be written 
in the form

$$
\begin{gathered}
\ldots \longrightarrow \operatorname{Ext}_{Q /(f)}^{p}(M, N) \longrightarrow \operatorname{Ext}_{Q^{\prime} /\left(f^{\prime}\right)}^{p}(M, N) \\
\longrightarrow \operatorname{Ext}_{Q /(f)}^{p-1}(M, N) \longrightarrow c
\end{gathered}
$$

Thus, if $\operatorname{Ext}_{Q /(f)}^{n}(M, N)=0$ for $n \gg 0$, then $\operatorname{Ext}_{Q^{\prime} /\left(f^{\prime}\right)}^{n}(M, N)=0$ for $n \gg 0$.

Conversely, if $\operatorname{Ext}_{Q^{\prime} /\left(f^{\prime}\right)}^{n}(M, N)=0$ for $n \gg 0$, then Proposition 5.12 shows that over the hypersurface $Q^{\prime \prime}=Q^{\prime} /\left(f^{\prime}\right)$ one of the modules $M$ or $N$ has finite projective dimension. Since $f^{\prime} \in \mathfrak{n}^{\prime 2}$ and $g^{\prime} \in \mathfrak{n}^{\prime} \backslash \mathfrak{n}^{\prime 2}$, we have $g \in \mathfrak{n}^{\prime \prime} \backslash \mathfrak{n}^{\prime \prime 2}$, where $\mathfrak{n}^{\prime \prime}=\mathfrak{n} Q^{\prime \prime}$. We conclude from 5.11 that $M$ or $N$ has finite projective dimension over $Q^{\prime} /\left(f^{\prime}, g^{\prime}\right) \cong Q /(f)$, so $\operatorname{Ext}_{Q /(f)}^{n}(M, N)=0$ for $n \gg 0$, as desired.

Proof of Theorem 5.6. By the definition of support varieties in 5.2, we may assume that $R$ is complete. We fix a Cohen presentation $\widehat{R} \cong Q /(\boldsymbol{f})$ with regular local ring $Q$ and regular sequence $\boldsymbol{f}=f_{1}, \ldots, f_{c}$; by Lemma 2.2, Theorem 5.5 and Theorem 5.3 we may assume that $k$ is algebraically closed. In $(\boldsymbol{f}) / \mathfrak{n}(\boldsymbol{f}) \cong k^{c}$ we set

$\mathrm{V}_{R}^{*}(M, N)=\mathrm{V}(Q, \boldsymbol{f} ; M, N) \quad$ and $\quad \mathrm{V}_{R}^{*}(M)=\mathrm{V}(Q, \boldsymbol{f} ; M, k)$.

(1) was noted in 2.1.

(2) $\operatorname{dim} \mathrm{V}_{R}^{*}(M, N)=\operatorname{cx}_{R}(M, N)$ by Proposition 2.4.2.

(3) Support varieties are homogeneous by (1), so $\mathrm{V}_{R}^{*}(M, N)=\{0\}$ if and only if $\operatorname{dim} \mathrm{V}_{R}^{*}(M, N)=0$. By (2) the latter condition is equivalent to $\operatorname{Ext}_{R}^{n}(M, N)=0$ for $n \gg 0$. Theorem 4.7 recasts the last condition as $\widehat{\operatorname{Ext}}_{R}^{n}(M, N)=0$ for $n \in \mathbb{Z}$.

(4) If $\operatorname{Ext}_{R}^{n}(M, N) \cong \operatorname{Ext}_{R}^{n+2}(M, N)$ for $n \gg 0$, then we can find a real number $c$ such that $\nu_{R}\left(\operatorname{Ext}_{R}^{n}(M, N)\right) \leq c$ for all $n \in \mathbb{Z}$, $\operatorname{socx}_{R}(M, N) \leq 1$.

Conversely, if $\operatorname{cx}_{R}(M, N) \leq 1$, then the graded module $\mathscr{E}=\operatorname{Ext}_{R}^{*}(M, N) \otimes_{R} k$ has dimension $\leq 1$ over $\mathcal{R}=\delta \otimes_{R} k$. Thus, we may find a linear combination $\chi=a_{1} \chi_{1}+\cdots+a_{c} \chi_{c}$ and an integer $i$ such that the map $\chi \otimes_{R} k: \varepsilon^{h} \rightarrow \varepsilon^{h+2}$ is bijective for $h \geq i$. By Nakayama's Lemma the map $\chi^{n}: \operatorname{Ext}_{R}^{h}(M, N) \rightarrow$ $\operatorname{Ext}_{R}^{h+2 n}(M, N)$ is surjective for $n \geq 0$. Choosing $j$ so that the sequence of graded $\&$-submodules

$$
\operatorname{Ker} \chi \subseteq \cdots \subseteq \operatorname{Ker} \chi^{n} \subseteq \operatorname{Ker} \chi^{n+2} \subseteq \cdots \subseteq \operatorname{Ext}_{R}^{*}(M, N)
$$

stabilizes for $n \geq j$, we see that the $R$-linear homomorphism $\chi: \operatorname{Ext}_{R}^{h}(M, N)$ $\rightarrow \operatorname{Ext}_{R}^{h+2}(M, N)$ is an isomorphism for $h \geq i+2 j$.

(5) Let $M^{\prime}, N^{\prime}$ be syzygies of $M, N$. As $\operatorname{Ext}_{R}^{i}(R, M)=0$ for $i>0$ and $\operatorname{Ext}_{R}^{i}(M, R)=0$ for $i>\operatorname{dim} R$, Proposition 2.4.3 yields $\mathrm{V}_{R}^{*}(M, N)=$ $\mathrm{V}_{R}^{*}\left(M^{\prime}, N^{\prime}\right)$. 
(7) If $M=\bigoplus_{i=1}^{r} M_{i}$ and $N=\bigoplus_{j=1}^{s} N_{j}$, then we have an isomorphism

$$
\operatorname{Ext}_{R}^{*}(M, N) \cong \bigoplus_{i, j} \operatorname{Ext}_{R}^{*}\left(M_{i}, N_{j}\right)
$$

of graded $R\left[\chi_{1}, \ldots, \chi_{c}\right]$-modules, hence $\mathrm{V}_{R}^{*}(M, N)=\bigcup_{i, j} \mathrm{~V}_{R}^{*}\left(M_{i}, N_{j}\right)$.

(8) By 2.6.4 and Corollary 2.7 we have $\mathrm{V}_{R}^{*}(M, N) \subseteq \mathrm{V}_{R}^{*}(M) \cap \mathrm{V}_{R}^{*}(N)$.

Assuming that equality fails, we may choose $\bar{f} \in \mathrm{V}_{R}^{*}(M) \cap \mathrm{V}_{R}^{*}(N) \subseteq$ $(\boldsymbol{f}) / \mathfrak{n}(\boldsymbol{f})$ with $\bar{f} \notin \mathrm{V}_{R}^{*}(M, N)$. If $f \in \mathfrak{n}$ is a lifting of $\bar{f}$, then proj $\operatorname{dim}_{Q /(f)} M$

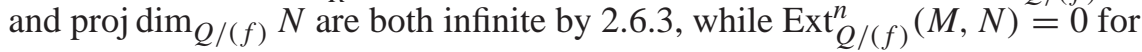
$n \gg 0$ by Theorem 2.5. This is ruled out by Proposition 5.12, so we conclude that $\mathrm{V}_{R}^{*}(M, N)=\mathrm{V}_{R}^{*}(M) \cap \mathrm{V}_{R}^{*}(N)$. Interchanging $M$ and $N$, we see that

$$
\mathrm{V}_{R}^{*}(N, M)=\mathrm{V}_{R}^{*}(N) \cap \mathrm{V}_{R}^{*}(M)=\mathrm{V}_{R}^{*}(M) \cap \mathrm{V}_{R}^{*}(N) .
$$
$\mathrm{V}_{R}^{*}(M)$.

(9) As $\mathrm{V}_{R}^{*}(k)=k^{c}$, (8) yields $\mathrm{V}_{R}^{*}(M, M)=\mathrm{V}_{R}^{*}(M, k)=\mathrm{V}_{R}^{*}(k, M)=$

(6) If $0 \rightarrow M_{1} \rightarrow M_{2} \rightarrow M_{3} \rightarrow 0$ is an exact sequence of $R$-modules, then by 1.1.2 the induced cohomology exact sequence of $R$-modules yields an exact sequence

$$
\begin{aligned}
& \operatorname{Ext}_{R}^{*}\left(M_{3}, k\right) \longrightarrow \operatorname{Ext}_{R}^{*}\left(M_{2}, k\right) \longrightarrow \operatorname{Ext}_{R}^{*}\left(M_{1}, k\right) \longrightarrow \\
& \operatorname{Ext}_{R}^{*}\left(M_{3}, k\right)[1] \longrightarrow \operatorname{Ext}_{R}^{*}\left(M_{2}, k\right)[1]
\end{aligned}
$$

of graded $\mathcal{R}$-modules, where brackets denote degree shifts. It follows that

$$
\mathrm{V}_{R}^{*}\left(M_{h}, k\right) \subseteq \mathrm{V}_{R}^{*}\left(M_{i}, k\right) \cup \mathrm{V}_{R}^{*}\left(M_{j}, k\right)
$$

for $\{h, i, j\}=\{1,2,3\}$. In view of (8), we then get

$$
\begin{aligned}
\mathrm{V}_{R}^{*}\left(M_{h}, N\right) & =\mathrm{V}_{R}^{*}\left(M_{h}\right) \cap \mathrm{V}_{R}^{*}(N) \\
& \subseteq\left(\mathrm{V}_{R}^{*}\left(M_{i}\right) \cup \mathrm{V}_{R}^{*}\left(M_{j}\right)\right) \cap \mathrm{V}_{R}^{*}(N) \\
& =\left(\mathrm{V}_{R}^{*}\left(M_{i}\right) \cap \mathrm{V}_{R}^{*}(N)\right) \cup\left(\mathrm{V}_{R}^{*}\left(M_{j}\right) \cap \mathrm{V}_{R}^{*}(N)\right) \\
& =\mathrm{V}_{R}^{*}\left(M_{i}, N\right) \cup \mathrm{V}_{R}^{*}\left(M_{j}, N\right) .
\end{aligned}
$$

Exact sequences $0 \rightarrow N_{1} \rightarrow N_{2} \rightarrow N_{3} \rightarrow 0$ are treated by a similar argument.

(10) If $M$ is Cohen-Macaulay of codimension $m$, then it is a perfect $Q$ module of grade $g=c+m$, so $\mathrm{V}_{R}^{*}(M)=\mathrm{V}_{R}^{*}\left(\operatorname{Ext}_{R}^{m}(M, R)\right)$ by Theorem 3.3.

\section{Vanishing of cohomology}

A ring $R$ is locally complete intersection, or l.c.i., if the local ring $R_{\mathfrak{m}}$ is a complete intersection for each maximal ideal $\mathfrak{m}$ of $R$.

As 1.c.i. rings are Gorenstein, each finite $R$-module has a complete resolution by 4.4.3 and 4.4.4. In particular, stable (co)homology is defined for any pair of finite modules. Its vanishing is described in the following result. 
6.1. Theorem. For finite modules $M, N$ over a locally complete intersection ring $R$ the following conditions are equivalent.

(i) $\widehat{\operatorname{Ext}}_{R}^{n}(M, N)=0$ for all $n \in \mathbb{Z}$.

(ii) $\widehat{\operatorname{Ext}}_{R}^{n}(N, M)=0$ for all $n \in \mathbb{Z}$.

(iii) $\widehat{\operatorname{Tor}}_{n}^{R}(M, N)=0$ for all $n \in \mathbb{Z}$.

(iv) $\mathrm{V}_{R_{\mathfrak{m}}}^{*}\left(M_{\mathfrak{m}}\right) \cap \mathrm{V}_{R_{\mathfrak{m}}}^{*}\left(N_{\mathfrak{m}}\right)=\{0\}$ for all $\mathfrak{m} \in \operatorname{Max}(R)$.

Proof. The conditions are local, so we may assume that $R$ is a local complete intersection with maximal ideal $\mathfrak{m}$. The equivalence of (i) or (ii) with (iv) is then established by parts (3) and (8) of Theorem 5.6, so it remains to deal with Tor's.

Choose a syzygy $M^{\prime}$ of $M$ that is also a syzygy in a complete resolution of $M$ and note that CI- $\operatorname{dim}_{R} M^{\prime}=0$ by 4.4.5 and 4.5.1. By 4.4.2 and 4.4.7 condition (iii) is equivalent to $\widehat{\operatorname{Ext}}_{R}^{n}\left(M^{*}, N\right)=0$ for all $n \in \mathbb{Z}$. By the already established equivalence of (i) and (iv) this condition is tantamount to $\mathrm{V}_{R}^{*}\left(M^{\prime *}\right) \cap \mathrm{V}_{R}^{*}(N)$ $=\{0\}$. Parts (10) and (5) of Theorem 5.6 translate the last condition into (iv). $\square$

Due to 4.4.6, the preceding result contains Theorem IV from the introduction.

To deduce Theorem III, we use the fact that the 1.c.i. property of $R$ is characterized by the finiteness of CI-dimension for all finite $R$-modules. For local rings this appears in [8]. The following global version is due to Liana Şega.

6.2. Proposition. A noetherian ring $R$ is locally complete intersection if and only if CI-dim $R$ is finite for each finite $R$-module $M$.

Proof. Recall from Sect. 4 that CI-dim $\operatorname{dim}_{R} M=\sup \left\{\right.$ CI-dim $\left.R_{\mathfrak{m}} M_{\mathfrak{m}} \mid \mathfrak{m} \in \operatorname{Max}(R)\right\}$.

If each finite $R$-module has finite CI-dimension, then CI-dim $R_{\mathfrak{m}} R_{\mathfrak{m}} / \mathfrak{m} R_{\mathfrak{m}}$ $<\infty$ for each maximal ideal $\mathfrak{m}$ of $R$, so $R_{\mathfrak{m}}$ is a local complete intersection by $[8,(1.3)]$.

Conversely, if $R$ is 1.c.i., then it is Gorenstein, so $\mathrm{G}-\operatorname{dim}_{R} M<\infty$ by 4.4.3. On the other hand, [3, (4.15)] yields G-dim $R=\sup \left\{\mathrm{G}-\operatorname{dim}_{R_{\mathfrak{m}}} M_{\mathfrak{m}} \mid \mathfrak{m} \in\right.$ $\operatorname{Max}(R)\}$. As CI-dim $R_{\mathfrak{m}} M_{\mathfrak{m}}=\mathrm{G}-\operatorname{dim}_{R_{\mathfrak{m}}} M_{\mathfrak{m}}$ by 4.5.1, we see that CI-dim $R$ is finite.

Proof of Theorem III. Let $R$ be a locally complete intersection ring, set $d=$ $\operatorname{dim} R$ and $c=\sup \left\{\operatorname{codim} R_{\mathfrak{m}} \mid \mathfrak{m} \in \operatorname{Max}(R)\right\}$, and let $M$ be a finite $R$-module.

For each $\mathfrak{m} \in \operatorname{Max}(R), 4.1 .2$ yields $\operatorname{cx}_{R_{\mathfrak{m}}} M_{\mathfrak{m}} \leq \operatorname{codim} R_{\mathfrak{m}}, \operatorname{socx}{ }_{R} M \leq c$. Also, CI-dim $R_{\mathfrak{m}} M_{\mathfrak{m}}=\operatorname{depth} R_{\mathfrak{m}}-\operatorname{depth}_{R_{\mathfrak{m}}} M_{\mathfrak{m}} \leq \operatorname{dim} R_{\mathfrak{m}}$ by 4.1 .5 , so CI- $\operatorname{dim}_{R} M \leq d$.

Putting together Theorems 4.7, 4.9, and 6.1 we get the desired equivalences. 
6.3. Remark. For a commutative noetherian ring $R$, consider the properties

(ee) if $\operatorname{Ext}_{R}^{n}(M, N)=0$ for $n \gg 0$, then $\operatorname{Ext}_{R}^{n}(N, M)=0$ for $n \gg 0$;

(et) if $\operatorname{Ext}_{R}^{n}(M, N)=0$ for $n \gg 0$, then $\operatorname{Tor}_{n}^{R}(M, N)=0$ for $n \gg 0$;

(te) if $\operatorname{Tor}_{n}^{R}(M, N)=0$ for $n \gg 0$, then $\operatorname{Ext}_{R}^{n}(M, N)=0$ for $n \gg 0$,

where $M, N$ range over all finite $R$-modules.

Denoting (lci) the condition that $R$ is locally complete intersection, and (gor) the one that it is Gorenstein, we have a diagram of implications

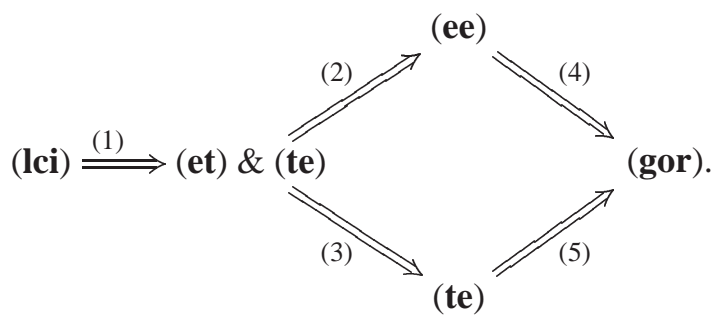

Indeed, (1) is a consequence of Theorem 6.1, via 4.4.6. The isomorphisms $\operatorname{Tor}_{n}^{R}(M, N) \cong \operatorname{Tor}_{n}^{R}(N, M)$ yield (2). To see (4) and (5), note that for each $\mathfrak{m} \in \operatorname{Max}(R)$ and all $n \in \mathbb{Z}$ we have $\operatorname{Ext}_{R_{\mathfrak{m}}}^{n}\left(R_{\mathfrak{m}} / \mathfrak{m} R_{\mathfrak{m}}, R_{\mathfrak{m}}\right) \cong$ $\operatorname{Ext}_{R}^{n}(R / \mathfrak{m}, R)_{\mathfrak{m}}$; the latter module vanishes for $n \gg 0$, and so $R$ is Goren-

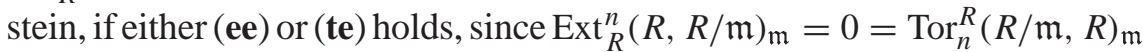
for $n>0$.

Forty years of research in commutative algebra have not produced a class of rings intermediate between locally complete intersections and Gorenstein rings, hence

6.4. Problem. Is any one of the implications in the diagram above reversible?

Acknowledgements. We thank Dan Grayson, Craig Huneke, Srikanth Iyengar, Dave Jorgensen, Liana Şega, and Roger Wiegand for interesting conversations on the subject of this paper.

\section{References}

1. T. Araya, Y. Yoshino, Remarks on a depth formula, a grade inequality, and a conjecture of Auslander, Comm. Algebra, 26 (1998), 3793-3806

2. M. Auslander, Modules over unramified regular local rings, Illinois J. Math. 5 (1961), 631-647

3. M. Auslander, M. Bridger, Stable module theory, Mem. Amer. Math. Soc. 94, Amer. Math. Soc., Providence, R.I., 1969

4. M. Auslander, S. Ding, Ø. Solberg, Liftings and weak liftings of modules, J. Algebra 156 (1993), 273-317 
5. L. L. Avramov, Modules of finite virtual projective dimension, Invent. math. 96 (1989), 71-101

6. L. L. Avramov, Infinite free resolutions, Six lectures on commutative algebra, Bellaterra 1996, Progr. Math. 166, Birkhäuser, Basel, 1998; pp. 1-118

7. L. L. Avramov, R.-O. Buchweitz, Homological algebra modulo a regular sequence with special attention to codimension two, J. Algebra (to appear)

8. L. L. Avramov, V. N. Gasharov, I. V. Peeva, Complete intersection dimension, Publ. Math. I.H.E.S. 86 (1997), 67-114

9. L. L. Avramov, L.-C. Sun, Cohomology operators defined by a deformation, J. Algebra 204 (1998), 684-710

10. D. J. Benson, Representations and cohomology: II. Cohomology of groups and modules, Cambridge Stud. Adv. Math. 31, Univ. Press, Cambridge, 1991

11. D. J. Benson, J. F. Carlson, G. R. Robinson, On the vanishing of group cohomology, J. Algebra 131 (1990), 40-73

12. N. Bourbaki, Algèbre commutative. IX: Anneaux locaux réguliers complets, Masson, Paris, 1983

13. R. O. Buchweitz, Maximal Cohen-Macaulay modules and Tate cohomology over Gorenstein rings, Preprint, University of Hannover, 1986

14. H. Cartan, S. Eilenberg, Homological algebra, Princeton Univ. Press, Princeton, NJ, 1956

15. J. Cornick, P. H. Kropholler, On complete resolutions, Topology Appl. 78 (1997), 235-250

16. D. Eisenbud, Homological algebra on a complete intersection, with an application to group representations, Trans. Amer. Math. Soc. 260 (1980), 35-64

17. E. M. Friedlander, A. Suslin, Cohomology of finite group schemes over a field, Invent. math. 127 (1997), 209-270

18. D. Grayson, M. Stillman, Macaulay 2, A software system for algebraic geometry and commutative algebra, available at http: / / www . math. uiuc.edu/Macaulay2

19. S. Goto, Vanishing of $\operatorname{Ext}_{A}^{i}(M, A)$, J. Math. Kyoto Univ. 22 (1982), 481-484

20. A. Grothendieck, Éléments de géométrie algèbrique. IV 4 , Publ. Math. I.H.E.S. 32, 1967

21. T. H. Gulliksen, A change of rings theorem, with applications to Poincaré series and intersection multiplicity, Math. Scand. 34 (1974), 167-183

22. C. Huneke, R. Wiegand, Tensor products of modules, rigidity and local cohomology, Math. Scand. 81 (1997), 161-183

23. D. A. Jorgensen, Tor and torsion on a complete intersection, J. Algebra 195 (1997), 526-537

24. D. A. Jorgensen, Complexity and Tor on a complete intersection, J. Algebra 211 (1999), 578-598

25. S. Lichtenbaum, On the vanishing of Tor in regular local rings, Illinois J. Math. 10 (1966), 220-226

26. V. Mehta, Endomorphisms of complexes and modules over Golod rings, Ph. D. Thesis, University of California, Berkeley, CA, 1976

27. C. Miller, Complexity of tensor products of modules and a theorem of Huneke-Wiegand, Proc. Amer. Math. Soc. 126 (1998), 53-60

28. M. P. Murthy, Modules over regular local rings, Illinois J. Math. 7 (1963), 558-565

29. M. Nagata, Local rings, Tracts Pure Appl. Math. 13, Wiley-Interscience, New York, 1962

30. D. Quillen, The spectrum of an equivariant cohomology ring. I, Ann. of Math. (2) 94 (1971), 549-572

31. J. Shamash, The Poincaré series of a local ring, J. Algebra 12 (1969), 453-470

32. J. Tate, Homology of Noetherian rings and of local rings, Illinois J. Math. 1 (1957), $14-25$ 